\title{
Macdonald Polynomials and Multivariable Basic Hypergeometric Series`
}

\author{
Michael J. SCHLOSSER
}

Fakultät für Mathematik, Universität Wien, Nordbergstraße 15, A-1090 Vienna, Austria

E-mail: michael.schlosser@univie.ac.at

URL: http://www.mat.univie.ac.at/ schlosse/

Received November 21, 2006; Published online March 30, 2007

Original article is available at http://www.emis.de/journals/SIGMA/2007/056/

\begin{abstract}
We study Macdonald polynomials from a basic hypergeometric series point of view. In particular, we show that the Pieri formula for Macdonald polynomials and its recently discovered inverse, a recursion formula for Macdonald polynomials, both represent multivariable extensions of the terminating very-well-poised ${ }_{6} \phi_{5}$ summation formula. We derive several new related identities including multivariate extensions of Jackson's verywell-poised ${ }_{8} \phi_{7}$ summation. Motivated by our basic hypergeometric analysis, we propose an extension of Macdonald polynomials to Macdonald symmetric functions indexed by partitions with complex parts. These appear to possess nice properties.
\end{abstract}

Key words: Macdonald polynomials; Pieri formula; recursion formula; matrix inversion; basic hypergeometric series; ${ }_{6} \phi_{5}$ summation; Jackson's ${ }_{8} \phi_{7}$ summation; $A_{n-1}$ series

2000 Mathematics Subject Classification: 33D52; 15A09; 33D67

\section{Introduction}

The objective of this paper is to study some aspects of $A_{n-1}$ Macdonald polynomials (which are a family of symmetric multivariable orthogonal polynomials associated with the irreducible reduced root system $A_{n-1}$, introduced by I.G. Macdonald [32] in the 1980's), with a particular emphasis on their connection to (multivariable) basic hypergeometric series.

Macdonald polynomials of type $A$ are indexed by integer partitions, and form a basis of the algebra of symmetric functions with rational coefficients in two parameters $q$ and $t$. They generalize many classical bases of this algebra, including monomial, elementary, Schur, HallLittlewood, and Jack symmetric functions. These particular cases correspond to various specializations of the indeterminates $q$ and $t$. In terms of basic hypergeometric series, the Macdonald polynomials correspond to a multivariable generalization of the continuous q-ultraspherical polynomials, see [25].

A principal tool for studying $q$-orthogonal polynomials (see e.g. [18]) is the theory of basic hypergeometric series (cf. [12]), rich of identities, having applications in different areas such as combinatorics, number theory, statistics, and physics (cf. [1]). Hypergeometric and basic hypergeometric series undoubtedly play a prominent role in special functions, see [2]. Even in one variable, they are still an object of active research. A notable recent advance includes elliptic (or modular) hypergeometric series (surveyed in [12, Ch. 11] and [52]) which is a one-parameter generalization of basic hypergeometric series, first introduced by Frenkel and Turaev [11] in a study related to statistical mechanics.

A convenient tool (suggested here) for further developing the theory of multivariable $q$ orthogonal polynomials is the theory of multivariable basic hypergeometric series associated with

${ }^{\star}$ This paper is a contribution to the Vadim Kuznetsov Memorial Issue 'Integrable Systems and Related Topics'. The full collection is available at http://www.emis.de/journals/SIGMA/kuznetsov.html 
root systems (or, equivalently, with Lie algebras). Basic hypergeometric series associated with the root system $A_{n-1}$ (or equivalently, associated with the unitary group $U(n)$ ) have their origin in the work of the three mathematical physicists Holman, Biedenharn, and Louck, starting in 1976, see $[16,17]$. Their work was done in the context of the quantum theory of angular momentum, using methods relying on the representation theory of the unitary group $U(n)$. Subsequently, extensive investigations in the theory of multiple basic hypergeometric series associated to the root system $A_{n-1}$ have been carried out by R.A. Gustafson, S.C. Milne, and later various other researchers. As result, many of the classical formulae for basic hypergeometric series from [12] have already been generalized to the setting of $A_{n-1}$ series (see Subsection 3.2 for some selected results).

An important result that connects $A_{n-1}$ basic hypergeometric series with Macdonald polynomials is Kajihara and Noumi's [22] explicit construction of raising operators of row type for Macdonald polynomials. Their construction utilized $A_{n-1}$ terminating ${ }_{1} \phi_{0}$ and ${ }_{2} \phi_{1}$ summations previously obtained by Milne [40] (which, however, were derived independently in [22] using Macdonald's $q$-difference operator).

In this paper we reveal yet more connections of $A_{n-1}$ basic hypergeometric series with Macdonald polynomials. On the other hand, we also understand the present work as a contribution towards the development of a theory of multivariable very-well-poised basic hypergeometric series involving Macdonald polynomials of type $A$.

Various identities for multiple basic hypergeometric series of Macdonald polynomial argument have been derived by Macdonald [33, p. 374, Eq. (4)], Kaneko [23, 24], Baker and Forrester [3], and Warnaar [54]. These authors in fact derived multivariable analogues of many of the classical summation and transformation formulae for basic hypergeometric series. As a matter of fact, none of these multivariate identities reduce to summations or transformations for very-wellpoised basic hypergeometric series in the univariate case. There are thus several other classical basic hypergeometric identities for which higher-dimensional extensions involving Macdonald polynomials of type $A$ have not yet been explicitly determined. In this paper we partly remedy this picture by explicitly pointing out several "Macdonald polynomial analogues" of very-wellpoised identities. Although some of these identities (such as the Pieri formula) are not new, their "very-well-poised context" appears so far to have kept unnoticed (at least, not explicitly mentioned in literature).

The present work makes some further ties between Macdonald polynomials and multiple basic hypergeometric series associated to $A_{n-1}$ apparent. In our investigations, we utilize some recent results from [31] and application of multidimensional inverse relations as main ingredients. Since the present paper can be regarded as a sequel to [31], it is appropriate to give a brief survey here describing those results of [31] which are relevant for this paper. This concerns, in particular, the derivation of a rather general explicit multidimensional matrix inverse [31, Th. 2.6]. The combination of a special case of this matrix inverse with the Pieri formula for Macdonald polynomials yielded a new recursion formula for Macdonald polynomials [31, Th. 4.1]. This formula, the main result of [31] - subsequently also derived by Lassalle [30] by alternative means, namely by functional equations - represents a recursion for the Macdonald polynomials $Q_{\lambda}$ on the row length of the indexing partition $\lambda$. More precisely, it expands a Macdonald polynomial of row length $n+1$ into products of one row and $n$ row Macdonald polynomials with explicitly determined coefficients (that are rational functions in $q$ and $t$ ). The formula is an $(n+1)$-variable extension of Jing and Józefiak's [20] well-known two-variable result and, as such, the Macdonald polynomial extension of the celebrated Jacobi-Trudi identity for Schur functions [33, p. 41, Eq. (3.4)].

In this paper we use special instances of the multidimensional matrix inverse of [31, Th. 2.6] and specific summation theorems for multivariable basic hypergeometric series to deduce several new identities for multivariable basic hypergeometric series. These turn out to play a special 
role (or, in some cases, we believe may be useful) when considering identities involving principally specialized Macdonald polynomials. In particular, we establish various new multivariable extensions of the terminating very-well-poised ${ }_{8} \phi_{7}$ summation theorem, and of (terminating and nonterminating) very-well-poised ${ }_{6} \phi_{5}$ summations. In one case, we provide (only) a conjectural multivariable terminating very-well-poised ${ }_{8} \phi_{7}$ summation, see Conjecture 4.5, which nevertheless we strongly believe to be true.

We further identify the Pieri formula for Macdonald polynomials and its inverse, the recursion formula for Macdonald polynomials, as two different multivariable generalizations of the terminating very-well-poised ${ }_{6} \phi_{5}$ summation. More precisely, this interpretation comes from the explicit structure the two formulae have after application of analytically continued principal specialization (coined "hypergeometric specialization" in the present context) to both sides of the respective identities.

Motivated by the basic hypergeometric analysis, we use the recursion formula to define $A_{n-1}$ Macdonald polynomials for "complex partitions", no longer indexed by sequences of non-increasing nonnegative integers but by arbitrary finite sequences of complex numbers. These $A_{n-1}$ Macdonald symmetric functions are identified as certain multivariable nonterminating basic hypergeometric series which appear to satisfy some nice properties. We only show a few of them here, in this point our exposition is mainly intended to hint towards specific new objects that we feel deserve future (more thorough and rigorous) study.

Our paper is organized as follows: In Section 2 we recollect some facts on symmetric functions, and, in particular, $A_{n-1}$ Macdonald polynomials. We conclude this section by stating the Pieri and the recursion formula, both playing crucial roles in the paper. In Section 3 we review some material on basic hypergeometric series in one and several variables, hereby explicitly listing several of the summations we need. In Section 4 we derive several identities of a new type that can be characterized by containing specific determinants in the summand. Section 5 is devoted to the hypergeometric specialization of identities involving $A_{n-1}$ Macdonald polynomials. Section 6 contains a brief discussion on more basic hypergeometric identities involving Macdonald polynomials. In Section 7 we consider Macdonald symmetric functions indexed by partitions with complex parts. Finally, the Appendix contains the multidimensional matrix inverse of [31], together with a number of useful corollaries.

\section{Preliminaries on $\boldsymbol{A}_{n-1}$ Macdonald polynomials}

Standard references for Macdonald polynomials are [32], [33, Ch. 6], and [34]. In the following, we recollect some facts we need.

Let $X=\left\{x_{1}, x_{2}, x_{3}, \ldots\right\}$ be an infinite set of indeterminates and $\mathcal{S}$ the algebra of symmetric functions of $X$ with coefficients in $\mathbb{Q}$. There are several standard algebraic bases of $\mathcal{S}$. Among these there are the power sum symmetric functions, defined by $p_{k}(X)=\sum_{i \geq 1} x_{i}^{k}$, for integer $k \geq 1$. Two other standard algebraic bases are the elementary and complete symmetric functions $e_{k}(X)$ and $h_{k}(X)$, which are defined, for integer $k \geq 0$, by their generating functions

$$
\prod_{i \geq 1}\left(1+u x_{i}\right)=\sum_{k \geq 0} u^{k} e_{k}(X), \quad \prod_{i \geq 1} \frac{1}{1-u x_{i}}=\sum_{k \geq 0} u^{k} h_{k}(X)
$$

A partition $\lambda=\left(\lambda_{1}, \lambda_{2}, \ldots\right)$ is a weakly decreasing (finite or infinite) sequence of nonnegative integers, with a finite number of positive integers, called parts. The number of positive parts is called the length of $\lambda$ and denoted $l(\lambda)$. If $l(\lambda)=n$, we often suppress any zeros appearing in the (sequential) representation of $\lambda$ and write $\lambda=\left(\lambda_{1}, \ldots, \lambda_{n}\right)$. For any integer $i \geq 1$, $m_{i}(\lambda)=\operatorname{card}\left\{j: \lambda_{j}=i\right\}$ is the multiplicity of $i$ in $\lambda$. Clearly $l(\lambda)=\sum_{i \geq 1} m_{i}(\lambda)$. We shall also 
write $\lambda=\left(1^{m_{1}}, 2^{m_{2}}, 3^{m_{3}}, \ldots\right)$ (where the parts now appear in increasing order). We set

$$
z_{\lambda}=\prod_{i \geq 1} i^{m_{i}(\lambda)} m_{i}(\lambda) !
$$

For any partition $\lambda$, the symmetric functions $e_{\lambda}, h_{\lambda}$ and $p_{\lambda}$ are defined by

$$
f_{\lambda}=\prod_{i=1}^{l(\lambda)} f_{\lambda_{i}}=\prod_{i \geq 1}\left(f_{i}\right)^{m_{i}(\lambda)}
$$

where $f_{i}$ stands for $e_{i}, h_{i}$, or $p_{i}$, respectively. (Here and in the following we sometimes omit writing out the argument $X$ of the function, for brevity, assuming there is no confusion.) These $e_{\lambda}, h_{\lambda}$, and $p_{\lambda}$ now each form a linear (vector space) basis of $\mathcal{S}$. Another classical basis is formed by the monomial symmetric functions $m_{\lambda}$, defined as the sum of all distinct monomials whose exponent is a permutation of $\lambda$.

For any two indeterminates $a, q$, we define the $q$-shifted factorial as

$$
(a ; q)_{\infty}=\prod_{j \geq 0}\left(1-a q^{j}\right), \quad \text { and } \quad(a ; q)_{k}=\frac{(a ; q)_{\infty}}{\left(a q^{k} ; q\right)_{\infty}}
$$

for arbitrary $k$ (usually an integer). Sometimes we rather consider expressions involving the $q$-shifted factorials in (2.2) in an analytic (holomorphic) sense. In such a case, $a, q$ are complex numbers, usually with $|q|<1$ (ensuring that the infinite product in (2.2) converges, a restriction not needed when $k$ is an integer and $(a ; q)_{k}$ is indeed a finite product) and $k$ can be any complex number. We also shall make frequent use of the condensed notation

$$
\left(a_{1}, \ldots, a_{m} ; q\right)_{k}=\left(a_{1} ; q\right)_{k} \cdots\left(a_{m} ; q\right)_{k}
$$

for arbitrary $k$ and nonnegative integer $m$.

Let $\mathbb{Q}(q, t)$ be the algebra of rational functions in the two indeterminates $q$, $t$, and Sym $=$ $\mathcal{S} \otimes \mathbb{Q}(q, t)$ the algebra of symmetric functions with coefficients in $\mathbb{Q}(q, t)$.

For any $k \geq 0$, the modified complete symmetric function $g_{k}(X ; q, t)$ is defined by the generating function

$$
\prod_{i \geq 1} \frac{\left(t u x_{i} ; q\right)_{\infty}}{\left(u x_{i} ; q\right)_{\infty}}=\sum_{k \geq 0} u^{k} g_{k}(X ; q, t) .
$$

It is often written in plethystic notation [29, p. 223], that is

$$
g_{k}(X ; q, t)=h_{k}\left[\frac{1-t}{1-q} X\right]
$$

The symmetric functions $g_{k}(q, t)$ form an algebraic basis of Sym. Their explicit expansion in terms of power sums and monomial symmetric functions has been given by Macdonald [33, pp. 311 and 314] and in terms of other classical bases by Lassalle [29, Sec. 10, p. 237]. The functions $g_{\lambda}(q, t)$, defined as in (2.1) and (2.4), form a linear basis of Sym.

We are ready to define the Macdonald polynomials $P_{\lambda}(X ; q, t)$. On one hand, they are of the form (recalling that $m_{\lambda}$ denotes a monomial symmetric function)

$P_{\lambda}(q, t)=m_{\lambda}+$ a linear combination of the $m_{\mu}$ for $\mu$ preceding $\lambda$ in lexicographical order. 
Furthermore, they form an orthogonal basis of Sym with respect to the scalar product $\langle,\rangle_{q, t}$ defined by

$$
\left\langle p_{\lambda}, p_{\mu}\right\rangle_{q, t}=\delta_{\lambda \mu} z_{\lambda} \prod_{i=1}^{l(\lambda)} \frac{1-q^{\lambda_{i}}}{1-t^{\lambda_{i}}} .
$$

Although these two conditions already overdetermine the symmetric functions $P_{\lambda}(X ; q, t)$, the latter can be shown to exist (and are moreover unique), see [33, p. 322].

Let $Q_{\lambda}(q, t)$ denote the dual basis of $P_{\lambda}(q, t)$ for this scalar product. These are also called Macdonald polynomials, they differ from the latter only by a rational function of $q$ and $t$. More precisely, one has

$$
Q_{\lambda}(X ; q, t)=b_{\lambda}(q, t) P_{\lambda}(X ; q, t),
$$

with $b_{\lambda}(q, t)=\left\langle P_{\lambda}(q, t), P_{\lambda}(q, t)\right\rangle_{q, t}{ }^{-1}$ specified as follows (see [33, p. 339, Eq. (6.19)] and [23, Prop. 3.2]):

$$
\begin{aligned}
b_{\lambda}(q, t) & =\prod_{1 \leq i \leq j \leq l(\lambda)} \frac{\left(q^{\lambda_{i}-\lambda_{j}} t^{j-i+1} ; q\right)_{\lambda_{j}-\lambda_{j+1}}}{\left(q^{\lambda_{i}-\lambda_{j}+1} t^{j-i} ; q\right)_{\lambda_{j}-\lambda_{j+1}}}=\prod_{1 \leq i \leq j \leq l(\lambda)} \frac{\left(q t^{j-i} ; q\right)_{\lambda_{i}-\lambda_{j}}\left(t^{j-i+1} ; q\right)_{\lambda_{i}-\lambda_{j+1}}}{\left(t^{j-i+1} ; q\right)_{\lambda_{i}-\lambda_{j}}\left(q t^{j-i} ; q\right)_{\lambda_{i}-\lambda_{j+1}}} \\
& =\prod_{i=1}^{n} \frac{\left(t^{n+1-i} ; q\right)_{\lambda_{i}}}{\left(q t^{n-i} ; q\right)_{\lambda_{i}}} \prod_{1 \leq i<j \leq n} \frac{\left(q t^{j-i}, t^{j-i} ; q\right)_{\lambda_{i}-\lambda_{j}}}{\left(t^{j-i+1}, q t^{j-i-1} ; q\right)_{\lambda_{i}-\lambda_{j}}},
\end{aligned}
$$

for any $n \geq l(\lambda)$. (It is easy to check that the latter expression indeed does not depend on $n$.)

The Macdonald polynomials factor nicely under "principal specialization" [33, p. 343, Example 5],

$$
P_{\lambda}\left(1, t, \ldots, t^{N-1} ; q, t\right)=t^{\sum_{i}(i-1) \lambda_{i}} \prod_{1 \leq i<j \leq N} \frac{\left(t^{j-i+1} ; q\right)_{\lambda_{i}-\lambda_{j}}}{\left(t^{j-i} ; q\right)_{\lambda_{i}-\lambda_{j}}} .
$$

A similar formula holds for principally specialized $Q_{\lambda}$, by combining (2.5), (2.6), and (2.7).

We mention two particular useful facts that hold in the case of a finite set of variables $X=\left\{x_{1}, \ldots, x_{n}\right\}$ (see [33, p. 323, Eq. (4.10), and p. 325, Eq. (4.17)]):

$$
P_{\lambda}\left(x_{1}, \ldots, x_{n} ; q, t\right)=0, \quad \text { if } \quad l(\lambda)>n,
$$

and

$$
P_{\left(\lambda_{1}, \lambda_{2}, \ldots, \lambda_{n}\right)}\left(x_{1}, \ldots, x_{n} ; q, t\right)=\left(x_{1} \ldots x_{n}\right)^{\lambda_{n}} P_{\left(\lambda_{1}-\lambda_{n}, \lambda_{2}-\lambda_{n}, \ldots, \lambda_{n-1}-\lambda_{n}, 0\right)}\left(x_{1}, \ldots, x_{n} ; q, t\right) .
$$

There exists [33, p. 327] an automorphism $\omega_{q, t}=\omega_{t, q}{ }^{-1}$ of Sym such that

$$
\omega_{q, t}\left(Q_{\lambda}(q, t)\right)=P_{\lambda^{\prime}}(t, q), \quad \omega_{q, t}\left(g_{k}(q, t)\right)=e_{k},
$$

with $\lambda^{\prime}$ the partition conjugate to $\lambda$, whose parts are given by $m_{k}\left(\lambda^{\prime}\right)=\lambda_{k}-\lambda_{k+1}$. In particular [33, p. 329, Eq. (5.5)], the Macdonald polynomials associated with a row or a column partition are given by

$$
\begin{array}{ll}
P_{1^{k}}(q, t)=e_{k}, & Q_{1^{k}}(q, t)=\frac{(t ; t)_{k}}{(q ; t)_{k}} e_{k}, \\
P_{(k)}(q, t)=\frac{(q ; q)_{k}}{(t ; q)_{k}} g_{k}(q, t), & Q_{(k)}(q, t)=g_{k}(q, t) .
\end{array}
$$

The parameters $q, t$ being kept fixed, we shall often write $P_{\mu}$ or $Q_{\mu}$ for $P_{\mu}(q, t)$ or $Q_{\mu}(q, t)$. 


\subsection{Pieri formula}

Let $u_{1}, \ldots, u_{n}$ be $n$ indeterminates and $\mathbb{N}$ the set of nonnegative integers. For $\theta=\left(\theta_{1}, \ldots, \theta_{n}\right) \in$ $\mathbb{N}^{n}$ let $|\theta|=\sum_{i=1}^{n} \theta_{i}$ and define

$$
d_{\theta_{1}, \ldots, \theta_{n}}^{(q, t)}\left(u_{1}, \ldots, u_{n}\right)=\prod_{k=1}^{n} \frac{\left(t, q^{|\theta|+1} u_{k} ; q\right)_{\theta_{k}}}{\left(q, q^{|\theta|} t u_{k} ; q\right)_{\theta_{k}}} \prod_{1 \leq i<j \leq n} \frac{\left(t u_{i} / u_{j}, q^{-\theta_{j}+1} u_{i} / t u_{j} ; q\right)_{\theta_{i}}}{\left(q u_{i} / u_{j}, q^{-\theta_{j}} u_{i} / u_{j} ; q\right)_{\theta_{i}}}
$$

The Macdonald polynomials satisfy a Pieri formula which generalizes the classical Pieri formula for Schur functions [33, p. 73, Eq. (5.16)]. This generalization was obtained by Macdonald [33, p. 331], and independently by Koornwinder [25].

Most of the time this Pieri formula is stated in combinatorial terms (as a sum over "horizontal strips"). In [31, Th. 3.1] it was formulated in "analytic" terms:

Theorem 2.1. Let $\lambda=\left(\lambda_{1}, \ldots, \lambda_{n}\right)$ be an arbitrary partition with length $\leq n$ and $\lambda_{n+1} \in \mathbb{N}$. Let $u_{i}=q^{\lambda_{i}-\lambda_{n+1}} t^{n-i}$, for $1 \leq i \leq n$. We have

$$
Q_{\left(\lambda_{1}, \ldots, \lambda_{n}\right)} Q_{\left(\lambda_{n+1}\right)}=\sum_{\theta \in \mathbb{N}^{n}} d_{\theta_{1}, \ldots, \theta_{n}}^{(q, t)}\left(u_{1}, \ldots, u_{n}\right) Q_{\left(\lambda_{1}+\theta_{1}, \ldots, \lambda_{n}+\theta_{n}, \lambda_{n+1}-|\theta|\right)} .
$$

The Pieri formula defines an infinite transition matrix. Indeed, let $\operatorname{Sym}(n+1)$ denote the algebra of symmetric polynomials in $n+1$ independent variables with coefficients in $\mathbb{Q}(q, t)$. Then [33, p. 313] the Macdonald polynomials $\left\{Q_{\lambda}, l(\lambda) \leq n+1\right\}$ form a basis of $\operatorname{Sym}(n+1)$, and so do the products $\left\{Q_{\mu} Q_{(m)}, l(\mu) \leq n, m \geq 0\right\}$.

\section{$2.2 \quad$ A recursion formula}

Again, let $u_{1}, \ldots, u_{n}$ be $n$ indeterminates. For $\theta=\left(\theta_{1}, \ldots, \theta_{n}\right) \in \mathbb{N}^{n}$ define

$$
\begin{aligned}
& c_{\theta_{1}, \ldots, \theta_{n}}^{(q, t)}\left(u_{1}, \ldots, u_{n}\right)=\prod_{i=1}^{n} t^{\theta_{i}} \frac{\left(q / t, q u_{k} ; q\right)_{\theta_{i}}}{\left(q, q t u_{i} ; q\right)_{\theta_{i}}} \prod_{1 \leq i<j \leq n} \frac{\left(q u_{i} / t u_{j}, q^{-\theta_{j}} t u_{i} / u_{j} ; q\right)_{\theta_{i}}}{\left(q u_{i} / u_{j}, q^{-\theta_{j}} u_{i} / u_{j} ; q\right)_{\theta_{i}}} \\
& \times \frac{1}{\prod_{1 \leq i<j \leq n}\left(q^{\theta_{i}} u_{i}-q^{\theta_{j}} u_{j}\right)} \operatorname{det}_{1 \leq i, j \leq n}\left[\left(q^{\theta_{i}} u_{i}\right)^{n-j}\left(1-t^{j-1} \frac{1-q^{\theta_{i}} t u_{i}}{1-q^{\theta_{i}} u_{i}} \prod_{s=1}^{n} \frac{u_{s}-q^{\theta_{i}} u_{i}}{t u_{s}-q^{\theta_{i}} u_{i}}\right)\right] .
\end{aligned}
$$

The following recursion formula for Macdonald polynomials was proved in [31, Th. 4.1] by inverting the Pieri formula in Theorem 2.1 utilizing a special case of the multidimensional matrix inverse in Corollary A.4.

Theorem 2.2. Let $\lambda=\left(\lambda_{1}, \ldots, \lambda_{n+1}\right)$ be an arbitrary partition with length $\leq n+1$. Let $u_{i}=q^{\lambda_{i}-\lambda_{n+1}} t^{n-i}$, for $1 \leq i \leq n$. We have

$$
Q_{\left(\lambda_{1}, \ldots, \lambda_{n+1}\right)}=\sum_{\theta \in \mathbb{N}^{n}} c_{\theta_{1}, \ldots, \theta_{n}}^{(q, t)}\left(u_{1}, \ldots, u_{n}\right) Q_{\left(\lambda_{n+1}-|\theta|\right)} Q_{\left(\lambda_{1}+\theta_{1}, \ldots, \lambda_{n}+\theta_{n}\right)} .
$$

In the case $n=1$, i.e. for partitions of length two, Theorem 2.2 reads

$$
Q_{\left(\lambda_{1}, \lambda_{2}\right)}=\sum_{\theta \in \mathbb{N}} c_{\theta}^{(q, t)}(u) Q_{\left(\lambda_{2}-\theta\right)} Q_{\left(\lambda_{1}+\theta\right)},
$$

with $u=q^{\lambda_{1}-\lambda_{2}}$ and

$$
c_{\theta}^{(q, t)}(u)=t^{\theta} \frac{(q / t, q u ; q)_{\theta}}{(q, q t u ; q)_{\theta}}\left(1-\frac{\left(1-t q^{\theta} u\right)}{\left(1-q^{\theta} u\right)} \frac{\left(u-q^{\theta} u\right)}{\left(t u-q^{\theta} u\right)}\right)
$$




$$
=t^{\theta} \frac{(q / t, q u ; q)_{\theta}}{(q, q t u ; q)_{\theta}} \frac{(t-1)}{\left(t-q^{\theta}\right)} \frac{\left(1-q^{2 \theta} u\right)}{\left(1-q^{\theta} u\right)}=t^{\theta} \frac{(1 / t, u ; q)_{\theta}}{(q, q t u ; q)_{\theta}} \frac{\left(1-q^{2 \theta} u\right)}{(1-u)} .
$$

This special case is due to Jing and Józefiak [20]. On the other hand, in the case $n=2$, i.e. for partitions of length three, Theorem 2.2 reduces to a formula stated by Lassalle [28].

Application of the automorphism $\omega_{q, t}$ to Theorem 2.2, while taking into account (2.10), gives the following equivalent result (cf. [31, Th. 4.2]).

Theorem 2.3. Let $\lambda=\left(1^{m_{1}}, 2^{m_{2}}, \ldots,(n+1)^{m_{n+1}}\right)$ be an arbitrary partition consisting of parts at most equal to $n+1$. Define $u_{i}=q^{n-i} t^{\sum_{j=i}^{n} m_{j}}$, for $1 \leq i \leq n+1$. We have

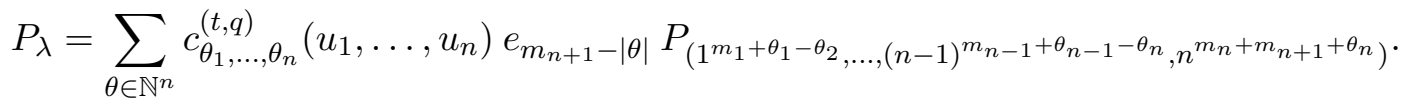

\section{Basic hypergeometric series}

\subsection{Classical (one-dimensional) basic hypergeometric series}

First we recall some standard notations for $q$-series and basic hypergeometric series (see Gasper and Rahman's text [12], for a standard reference). In the following we shall consider $q$ a (fixed) complex parameter with $0<|q|<1$. Further, for a complex parameter $a$, we use the definition of the $q$-shifted factorial as given in (2.2) and (2.3), for any complex number $k$.

The basic hypergeometric ${ }_{r} \phi_{r-1}$ series with upper parameters $a_{1}, \ldots, a_{r}$, lower parameters $b_{1}, \ldots, b_{r-1}$, base $q$, and argument $z$ is defined as follows:

$$
{ }_{r} \phi_{r-1}\left[\begin{array}{c}
a_{1}, a_{2}, \ldots, a_{r} \\
b_{1}, b_{2}, \ldots, b_{r-1}
\end{array} ; q, z\right]=\sum_{k=0}^{\infty} \frac{\left(a_{1}, a_{2}, \ldots, a_{r} ; q\right)_{k}}{\left(q, b_{1}, \ldots, b_{r-1} ; q\right)_{k}} z^{k}
$$

An ${ }_{r} \phi_{r-1}$ series terminates if one of its upper parameters is of the form $q^{-m}$ with $m=$ $0,1,2, \ldots$, because

$$
\left(q^{-m} ; q\right)_{k}=0, \quad k=m+1, m+2, \ldots
$$

The ${ }_{r} \phi_{r-1}$ series, if it does not terminate, converges absolutely for $|z|<1$.

In our computations throughout this paper, we make frequent use of some elementary identities for $q$-shifted factorials, listed in [12, Appendix I]. We list a few important summation theorems from [12, Appendix II], for quick reference.

We start with the (nonterminating) q-binomial theorem (cf. [12, Eq. (II.2)])

$$
{ }_{1} \phi_{0}\left[\begin{array}{l}
a \\
-
\end{array} ;, z\right]=\frac{(a z ; q)_{\infty}}{(z ; q)_{\infty}}, \quad|z|<1,
$$

an identity first discovered by Cauchy [8] in 1843 .

Further, we have the $q$-Gauß summation (cf. [12, Eq. (II.8)]),

$$
{ }_{2} \phi_{1}\left[\begin{array}{c}
a, b \\
c
\end{array} ;, \frac{c}{a b}\right]=\frac{(c / a, c / b ; q)_{\infty}}{(c, c / a b ; q)_{\infty}}, \quad|c / a b|<1
$$

which is due to Heine [15].

An identity of fundamental importance is the terminating ${ }_{6} \phi_{5}$ summation theorem (cf. [12, Eq. (II.21)])

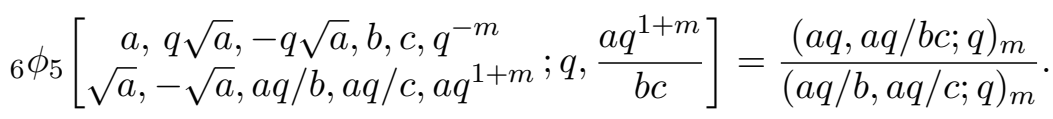


This identity can be extended (by analytic continuation) to Rogers' [47] nonterminating ${ }_{6} \phi_{5}$ summation theorem (cf. [12, Eq. (II.20)])

$$
{ }_{6} \phi_{5}\left[\begin{array}{c}
a, q \sqrt{a},-q \sqrt{a}, b, c, d \\
\sqrt{a},-\sqrt{a}, a q / b, a q / c, a q / d
\end{array} ; q, \frac{a q}{b c d}\right]=\frac{(a q, a q / b c, a q / b d, a q / c d ; q)_{\infty}}{(a q / b, a q / c, a q / d, a q / b c d ; q)_{\infty}},
$$

where $|a q / b c d|<1$. Clearly, (3.4) reduces to (3.3) for $d=q^{-m}$.

The series in (3.3) and (3.4) both are examples of so-called "very-well-poised" series. Another example is Jackson's [19] terminating very-well-poised ${ }_{8} \phi_{7}$ summation (cf. [12, Eq. (II.22)]),

$$
{ }_{8} \phi_{7}\left[\begin{array}{c}
a, q \sqrt{a},-q \sqrt{a}, b, c, d, a^{2} q^{1+n} / b c d, q^{-n} \\
\sqrt{a},-\sqrt{a}, a q / b, a q / c, a q / d, b c d q^{-n} / a, a q^{1+n} ; q, q
\end{array}\right]=\frac{(a q, a q / b c, a q / b d, a q / c d ; q)_{n}}{(a q / b, a q / c, a q / d, a q / b c d ; q)_{n}},
$$

which contains (3.4) as a special case $(n \rightarrow \infty)$. (The series in (3.5) is even "balanced" but we do not need this notion here.) Jackson's summation (3.5) stands on the top of the classical hierarchy of summation theorems for very-well-poised basic hypergeometric series.

An ${ }_{r} \phi_{r-1}$ series is well-poised if the parameters satisfy the relations

$$
q a_{1}=a_{2} b_{1}=a_{3} b_{2}=\cdots=a_{r} b_{r-1} .
$$

It is very-well-poised if, in addition, $a_{2}=q \sqrt{a_{1}}, a_{3}=-q \sqrt{a_{1}}$. Note that

$$
\frac{\left(q \sqrt{a_{1}},-q \sqrt{a_{1}} ; q\right)_{k}}{\left(\sqrt{a_{1}},-\sqrt{a_{1}} ; q\right)_{k}}=\frac{1-a_{1} q^{2 k}}{1-a_{1}}
$$

appears as a factor in the summand of a very-well-poised series. The parameter $a_{1}$ is usually referred to as the special parameter of such a series, and we call (3.6) the very-well-poised term of the series.

\section{$3.2 \quad A_{n-1}$ basic hypergeometric series}

For convenience, we employ here (and elsewhere) the notation $|\mathbf{k}|=k_{1}+\cdots+k_{n}$, where $\mathbf{k}=$ $\left(k_{1}, \ldots, k_{n}\right)$. We recall the following fundamental result from [35, Th. 1.49].

Proposition 3.1 (Milne). Let $a_{1}, \ldots, a_{n}$, and $u_{1}, \ldots, u_{n}$ be indeterminate, let $M$ be a nonnegative integer. Then

$$
\sum_{\substack{k_{1}, \ldots, k_{n} \geq 0 \\|\mathbf{k}|=M}} \prod_{\substack{\leq \leq i<j \leq n \\ u_{i}-u_{j}}} \frac{u_{i} q^{k_{i}}-u_{j} q^{k_{j}}}{u_{i, j=1}^{n}} \frac{\left(a_{j} u_{i} / u_{j} ; q\right)_{k_{i}}}{\left(q u_{i} / u_{j} ; q\right)_{k_{i}}}=\frac{\left(a_{1} a_{2} \ldots a_{n} ; q\right)_{M}}{(q ; q)_{M}} .
$$

The $n=2$ case of (3.7) is equivalent to (3.3). Proposition 3.1 played an important role in Milne's elementary derivation of the Macdonald identities for the affine Lie algebra $A_{n}^{(1)}$. It further serves as a starting point in Milne's approach to $A_{n-1}$ or $U(n)$ series, see [41]. Its original proof made use of a particular $q$-difference equation, and induction. An even simpler inductive proof of Proposition 3.1 was recently discovered by Rosengren [48, Sec. 2] (see also [12, Sec. 11.7]).

An immediate consequence of Proposition 3.1 is the following $n$-dimensional extension of (3.1), first given in [35, Th. 1.47].

Proposition 3.2 ((Milne) An $A_{n-1}$ nonterminating $q$-binomial theorem). Let $a_{1}, \ldots, a_{n}$, $z$, and $u_{1}, \ldots, u_{n}$ be indeterminate. Then

$$
\sum_{k_{1}, \ldots, k_{n}=0}^{\infty} \prod_{1 \leq i<j \leq n} \frac{u_{i} q^{k_{i}}-u_{j} q^{k_{j}}}{u_{i}-u_{j}} \prod_{i, j=1}^{n} \frac{\left(a_{j} u_{i} / u_{j} ; q\right)_{k_{i}}}{\left(q u_{i} / u_{j} ; q\right)_{k_{i}}} \cdot z^{|\mathbf{k}|}=\frac{\left(a_{1} \ldots a_{n} z ; q\right)_{\infty}}{(z ; q)_{\infty}}
$$

provided $|z|<1$. 
Clearly, Proposition 3.2 follows from Proposition 3.1 by multiplying both sides of (3.7) by $z^{M}$ and summing over all $M \geq 0$. The right-hand side is then simplified using (3.1).

In the above cases, we have

$$
\prod_{1 \leq i<j \leq n} \frac{u_{i} q^{k_{i}}-u_{j} q^{k_{j}}}{u_{i}-u_{j}}
$$

appearing as a factor of the summand. Since we may associate (3.8) with the product side of the Weyl denominator formula for the root system $A_{n-1}$ (see e.g. D. Stanton [53]), we call such a series $A_{n-1}$ basic hypergeometric series. Note that often in the literature these $n$-dimensional series are called $A_{n}$ series instead of $A_{n-1}$ series.

Note that on the left-hand side of (3.10), e.g., we have, in addition to (3.8),

$$
\prod_{i=1}^{n} \frac{1-a u_{i} q^{k_{i}+|\mathbf{k}|}}{1-a u_{i}}
$$

appearing as a factor in the summand of the series. It is easy to see that the $n=1$ case of (3.9) essentially reduces to (3.6). We call the product of (3.8) and (3.9) the very-well-poised term of the multiple series. To clarify the special appearance of the very-well-poised term (even in the one-dimensional case), it is useful to view the series in one higher dimension. In particular, we can write

$$
\prod_{1 \leq i<j \leq n} \frac{u_{i} q^{k_{i}}-u_{j} q^{k_{j}}}{u_{i}-u_{j}} \prod_{i=1}^{n} \frac{1-a u_{i} q^{k_{i}+|\mathbf{k}|}}{1-a u_{i}}=q^{|\mathbf{k}|} \prod_{1 \leq i<j \leq n+1} \frac{u_{i} q^{k_{i}}-u_{j} q^{k_{j}}}{u_{i}-u_{j}},
$$

where $u_{n+1}=1 / a$ and $k_{n+1}=-|\mathbf{k}|$. Indeed, some $A_{n-1}$ basic hypergeometric series identities are sometimes better viewed as identities associated to the affine root system $\tilde{A}_{n}$ (or, equivalently, the special unitary group $S U(n+1))$.

Multidimensional basic hypergeometric series associated with the root system $A_{n-1}$ (or equivalently, associated with the unitary group $U(n)$ ) have their origin in the work of the three mathematical physicists Holman, Biedenharn, and Louck, see [16, 17]. Their work was done in the context of the quantum theory of angular momentum, using methods relying on the representation theory of the unitary group $U(n)$. Subsequently, extensive investigations in the theory of multiple basic hypergeometric series associated with the root system $A_{n-1}$ have been carried out by R.A. Gustafson, S.C. Milne, and several other researchers. As result, many of the classical formulae for basic hypergeometric series from [12] have already been generalized to the setting of $A_{n-1}$ series, see e.g. the references [5, 10, 13, 14, 35, 36, 38, 39, 40, 42, 43, 49].

An immediate consequence of Proposition 3.1 is the following extension of (3.3), a result first obtained in [36, Th. 1.38].

Proposition 3.3 ((Milne) An $A_{n-1}$ terminating ${ }_{6} \phi_{5}$ summation). Let $a, b, c_{1}, \ldots, c_{n}$, and $u_{1}, \ldots, u_{n}$ be indeterminate, let $M$ be a nonnegative integer. Then

$$
\begin{aligned}
& \sum_{\substack{k_{1}, \ldots, k_{n} \geq 0 \\
|\mathbf{k}| \leq M}} \prod_{1 \leq i<j \leq n} \frac{u_{i} q^{k_{i}}-u_{j} q^{k_{j}}}{u_{i}-u_{j}} \prod_{i=1}^{n} \frac{1-a u_{i} q^{k_{i}+|\mathbf{k}|}}{1-a u_{i}} \prod_{i=1}^{n} \frac{\left(a u_{i} ; q\right)_{|\mathbf{k}|}}{\left(a u_{i} q / c_{i} ; q\right)_{|\mathbf{k}|}} \\
& \quad \times \prod_{i, j=1}^{n} \frac{\left(c_{j} u_{i} / u_{j} ; q\right)_{k_{i}}}{\left(q u_{i} / u_{j} ; q\right)_{k_{i}}} \prod_{i=1}^{n} \frac{\left(b u_{i} ; q\right)_{k_{i}}}{\left(a u_{i} q^{1+M} ; q\right)_{k_{i}}} \cdot \frac{\left(q^{-M} ; q\right)_{|\mathbf{k}|}}{(a q / b ; q)_{|\mathbf{k}|}}\left(\frac{a q^{1+M}}{b c_{1} \cdots c_{n}}\right)^{|\mathbf{k}|} \\
& =\frac{\left(a q / b c_{1} \cdots c_{n} ; q\right)_{M}}{(a q / b ; q)_{M}} \prod_{i=1}^{n} \frac{\left(a u_{i} q ; q\right)_{M}}{\left(a u_{i} q / c_{i} ; q\right)_{M}} .
\end{aligned}
$$


Proof (see e.g. [4]). To derive Proposition 3.3 from Proposition 3.1, just replace $n$ by $n+1$ in (3.7), and replace $k_{n+1}$ by $M-|\mathbf{k}|$, so that in the resulting sum the summation index ranges over the $n$-tetrahedron $0 \leq|\mathbf{k}| \leq M$. Finally, suitably relabel parameters to obtain (3.10).

Proposition 3.3 can be extended by analytic continuation to the following multivariable generalization of (3.4), a result first obtained in [36, Th. 1.44].

Proposition 3.4 ((Milne) An $A_{n-1}$ nonterminating ${ }_{6} \phi_{5}$ summation). Let $a, b, c_{1}, \ldots, c_{n}$, $d$, and $u_{1}, \ldots, u_{n}$ be indeterminate. Then

$$
\begin{aligned}
& \sum_{k_{1}, \ldots, k_{n} \geq 0} \prod_{1 \leq i<j \leq n} \frac{u_{i} q^{k_{i}}-u_{j} q^{k_{j}}}{u_{i}-u_{j}} \prod_{i=1}^{n} \frac{1-a u_{i} q^{k_{i}+|\mathbf{k}|}}{1-a u_{i}} \prod_{i=1}^{n} \frac{\left(a u_{i} ; q\right)_{|\mathbf{k}|}}{\left(a u_{i} q / c_{i} ; q\right)_{|\mathbf{k}|}} \\
& \quad \times \prod_{i, j=1}^{n} \frac{\left(c_{j} u_{i} / u_{j} ; q\right)_{k_{i}}}{\left(q u_{i} / u_{j} ; q\right)_{k_{i}}} \prod_{i=1}^{n} \frac{\left(b u_{i} ; q\right)_{k_{i}}}{\left(a u_{i} q / d ; q\right)_{k_{i}}} \cdot \frac{(d ; q)_{|\mathbf{k}|}}{(a q / b ; q)_{|\mathbf{k}|}}\left(\frac{a q}{b c_{1} \cdots c_{n} d}\right)^{|\mathbf{k}|} \\
& =\frac{\left(a q / b c_{1} \cdots c_{n}, a q / b d ; q\right)_{\infty}}{\left(a q / b, a q / b c_{1} \cdots c_{n} d ; q\right)_{\infty}} \prod_{i=1}^{n} \frac{\left(a u_{i} q, a u_{i} q / c_{i} d ; q\right)_{\infty}}{\left(a u_{i} q / c_{i}, a u_{i} q / d ; q\right)_{\infty}}
\end{aligned}
$$

provided $\left|a q / b c_{1} \cdots c_{n} d\right|<1$.

Clearly, Proposition 3.4 reduces to Proposition 3.3 for $d=q^{-M}$.

Proof. Both sides of (3.11) are analytic in $1 / d$ in a domain $\mathcal{D}$ around the origin. Now, if $1 / d=q^{M}$ the formula holds due to Proposition 3.3 , for all $M=0,1,2, \ldots$. Since the infinite sequence $q^{M}$ has an accumulation point, namely 0 , in the domain of analyticity of $1 / d$, we can apply the identity theorem to deduce that $(3.11)$ is true for all $1 / d$ throughout $\mathcal{D}$. By analytic continuation the identity holds for all $1 / d$ in its region of convergence, i.e. in the disc $|1 / d|<\left|b c_{1} \cdots c_{n} / a q\right|$.

We will need the following identity from [40, Th. 7.6] which can be obtained from Proposition 3.4 by letting $b \mapsto a q / c$, followed by $a \rightarrow 0$, and the subsequent substitutions $c_{i} \mapsto a_{i}$, $i=1, \ldots, n$, and $d \mapsto b$. It represents a multivariable generalization of the $q$-Gauß summation in $(3.2)$.

Proposition 3.5 ((Milne) An $A_{n-1}$ nonterminating ${ }_{2} \phi_{1}$ summation). Let $a_{1}, \ldots, a_{n}, b$, $c$, and $u_{1}, \ldots, u_{n}$ be indeterminate. Then

$$
\begin{aligned}
& \sum_{k_{1}, \ldots, k_{n} \geq 0} \prod_{1 \leq i<j \leq n} \frac{u_{i} q^{k_{i}}-u_{j} q^{k_{j}}}{u_{i}-u_{j}} \prod_{i, j=1}^{n} \frac{\left(a_{j} u_{i} / u_{j} ; q\right)_{k_{i}}}{\left(q u_{i} / u_{j} ; q\right)_{k_{i}}} \cdot \frac{(b ; q)_{|\mathbf{k}|}}{(c ; q)_{|\mathbf{k}|}}\left(\frac{c}{a_{1} \cdots a_{n} b}\right)^{|\mathbf{k}|} \\
& =\frac{\left(c / a_{1} \cdots a_{n}, c / b ; q\right)_{\infty}}{\left(c, c / a_{1} \cdots a_{n} b ; q\right)_{\infty}}
\end{aligned}
$$

provided $\left|c / a_{1} \cdots a_{n} b\right|<1$.

The following identity in Proposition 3.6 is even more general than Propositions 3.3 and 3.4. It was first obtained in [37, Th. 6.17]. A simple inductive proof of Proposition 3.6 was recently discovered by Rosengren [48, Sec. 5] (see also [12, Sec. 11.7]).

Proposition 3.6 ((Milne) An $A_{n-1}$ terminating ${ }_{8} \phi_{7}$ summation). Let $a, b, c_{1}, \ldots, c_{n}, d$, and $u_{1}, \ldots, u_{n}$ be indeterminate, let $M$ be a nonnegative integer. Then

$$
\sum_{\substack{k_{1}, \ldots, k_{n} \geq 0 \\|\mathbf{k}| \leq M}} \prod_{\substack{\leq \leq i<j \leq n \\ u_{i}-u_{j}}} \frac{u_{i} q^{k_{i}}-u_{j} q^{k_{j}}}{u_{i=1}} \prod^{n} \frac{1-a u_{i} q^{k_{i}+|\mathbf{k}|}}{1-a u_{i}} \prod_{i=1}^{n} \frac{\left(a u_{i} ; q\right)_{|\mathbf{k}|}}{\left(a u_{i} q / c_{i} ; q\right)_{|\mathbf{k}|}} \prod_{i, j=1}^{n} \frac{\left(c_{j} u_{i} / u_{j} ; q\right)_{k_{i}}}{\left(q u_{i} / u_{j} ; q\right)_{k_{i}}}
$$




$$
\begin{aligned}
& \times \prod_{i=1}^{n} \frac{\left(b u_{i}, a^{2} u_{i} q^{1+M} / b c_{1} \cdots c_{n} d ; q\right)_{k_{i}}}{\left(a u_{i} q / d, a u_{i} q^{1+M} ; q\right)_{k_{i}}} \cdot \frac{\left(d, q^{-M} ; q\right)_{|\mathbf{k}|}}{\left(a q / b, b c_{1} \cdots c_{n} d q^{-M} / a ; q\right)_{|\mathbf{k}|}} q^{|\mathbf{k}|} \\
& =\frac{\left(a q / b d, a q / b c_{1} \cdots c_{n} ; q\right)_{M}}{\left(a q / b, a q / b c_{1} \cdots c_{n} d ; q\right)_{M}} \prod_{i=1}^{n} \frac{\left(a u_{i} q, a u_{i} q / c_{i} d ; q\right)_{M}}{\left(a u_{i} q / c_{i}, a u_{i} q / d ; q\right)_{M}}
\end{aligned}
$$

For convenience, we also list the following identity which is not of $A_{n-1}$ but of $C_{n}$ type. It was derived in [10, Th. 4.1], and, independently, in [42, Th. 6.13].

Proposition 3.7 ((Denis-Gustafson; Milne-Lilly) A $C_{n}$ terminating ${ }_{8} \phi_{7}$ summation). Let $a, b, c, d$ and $u_{1}, \ldots, u_{n}$ be indeterminate, let $m_{1}, \ldots, m_{n}$ be nonnegative integers. Then

$$
\begin{aligned}
& \sum_{\substack{0 \leq k_{i} \leq m_{i} \\
i=1, \ldots, n}} \prod_{1 \leq i<j \leq n} \frac{u_{i} q^{k_{i}}-u_{j} q^{k_{j}}}{u_{i}-u_{j}} \prod_{1 \leq i \leq j \leq n} \frac{1-a u_{i} u_{j} q^{k_{i}+k_{j}}}{1-a u_{i} u_{j}} \prod_{i, j=1}^{n} \frac{\left(q^{-m_{j}} u_{i} / u_{j}, a u_{i} u_{j} ; q\right)_{k_{i}}}{\left(a u_{i} u_{j} q^{1+m_{j}}, q u_{i} / u_{j} ; q\right)_{k_{i}}} \\
& \quad \times \prod_{i=1}^{n} \frac{\left(b u_{i}, c u_{i}, d u_{i}, a^{2} u_{i} q^{1+|\mathbf{m}|} / b c d ; q\right)_{k_{i}}}{\left(a u_{i} q / b, a u_{i} q / c, a u_{i} q / d, b c d u_{i} q^{-|\mathbf{m}| / a ; q)_{k_{i}}} \cdot q^{|\mathbf{k}|}\right.} \\
& =\prod_{1 \leq i<j \leq n}\left(a u_{i} u_{j} q ; q\right)_{m_{i}+m_{j}}^{-1} \prod_{i, j=1}^{n}\left(a u_{i} u_{j} q ; q\right)_{m_{i}} \\
& \quad \times \frac{(a q / b c, a q / b d, a q / c d ; q)_{|\mathbf{m}|}}{\prod_{i=1}^{n}\left(a u_{i} q / b, a u_{i} q / c, a u_{i} q / d, a q^{\left.1+|\mathbf{m}|-m_{i} / b c d u_{i} ; q\right)_{m_{i}}}\right.}
\end{aligned}
$$

\section{Multivariable basic hypergeometric series of a new type}

Here we derive several new summations for multivariable basic hypergeometric series of a new type. Their special feature is that the series involve specific determinants in the summand. The first result is obtained from the $A_{n-1}{ }_{8} \phi_{7}$ summation in Proposition 3.6, combined with the multivariable matrix inverse in Corollary A.4.

Theorem 4.1 (A multivariable terminating ${ }_{8} \phi_{7}$ summation). Let $b, d, t_{0}, t_{1}, \ldots, t_{n}$, $u_{1}, \ldots, u_{n}$ be indeterminate, let $M$ be a nonnegative integer. Then

$$
\begin{aligned}
& \sum_{\substack{k_{1}, \ldots, k_{n} \geq 0 \\
|\mathbf{k}| \leq M}} \prod_{i, j=1}^{n} \frac{\left(q u_{i} / t_{i} u_{j} ; q\right)_{k_{i}}}{\left(q u_{i} / u_{j} ; q\right)_{k_{i}}} \prod_{1 \leq i<j \leq n} \frac{\left(t_{j} u_{i} / u_{j} ; q\right)_{k_{i}-k_{j}}}{\left(q u_{i} / t_{i} u_{j} ; q\right)_{k_{i}-k_{j}}} \prod_{1 \leq i<j \leq n} \frac{1}{u_{i}-u_{j}} \\
& \quad \times \operatorname{det}_{1 \leq i, j \leq n}\left[( u _ { i } q ^ { k _ { i } } ) ^ { n - j } \left(1-t_{i}^{j-n-1} \frac{1-t_{0} u_{i} q^{k_{i}}}{1-t_{0} u_{i} q^{k_{i}} / t_{i}} \prod_{s=1}^{n} \frac{u_{i} q^{k_{i}}-u_{s}}{\left.\left.u_{i} q^{k_{i} / t_{i}-u_{s}}\right)\right]}\right.\right. \\
& \quad \times \frac{\left(d, q^{-M} ; q\right)_{|\mathbf{k}|}}{\left(b d q^{-M} / t_{0}, t_{0} q / b t_{1} \cdots t_{n} ; q\right)_{|\mathbf{k}|}} \prod_{i=1}^{n} \frac{\left(t_{0} u_{i} q / t_{i}, b u_{i}, t_{0}^{2} u_{i} q^{1+M} / b d t_{1} \cdots t_{n} ; q\right)_{k_{i}}}{\left(t_{0} u_{i} q, t_{0} u_{i} q / d t_{i}, t_{0} u_{i} q^{1+M} / t_{i} ; q\right)_{k_{i}}} \\
& \quad \times \prod_{i=1}^{n} \frac{\left(d q^{-M} / t_{0} u_{i} ; q\right)_{|\mathbf{k}|-k_{i}}}{\left(d t_{i} q^{-M} / t_{0} u_{i} ; q\right)_{|\mathbf{k}|-k_{i}}} \cdot q^{|\mathbf{k}|+\sum_{i=1}^{n}(1-i) k_{i}} \prod_{i=1}^{n} t_{i}(i-1) k_{i}+\sum_{j=i+1}^{n} k_{j} \\
& =\frac{\left(t_{0} q / b, t_{0} q / b d t_{1} \cdots t_{n} ; q\right)_{M}}{\left(t_{0} q / b d, t_{0} q / b t_{1} \cdots t_{n} ; q\right)_{M}} \prod_{i=1}^{n} \frac{\left(t_{0} u_{i} q / t_{i}, t_{0} u_{i} q / d ; q\right)_{M}}{\left(t_{0} u_{i} q, t_{0} u_{i} q / d t_{i} ; q\right)_{M}}
\end{aligned}
$$


Proof. We apply multidimensional inverse relations. We have (A.4b) by the $a \mapsto t_{0} q^{|\mathbf{I}|}, c_{i} \mapsto t_{i}$, $d \mapsto d q^{|\mathbf{l}|}, u_{i} \mapsto u_{i} q^{l_{i}}, i=1, \ldots, n$, and $M \mapsto M-|\mathbf{l}|$ case of Proposition 3.6, where

$$
\begin{aligned}
b_{\mathbf{k}}= & \prod_{1 \leq i<j \leq n}\left(u_{i} q^{k_{i}}-u_{j} q^{k_{j}}\right) \prod_{i, j=1}^{n} \frac{\left(t_{j} u_{i} / u_{j} ; q\right)_{k_{i}-k_{j}}}{\left(q u_{i} / u_{j} ; q\right)_{k_{i}-k_{j}}} \prod_{i=1}^{n} \frac{\left(t_{0} u_{i} q ; q\right)_{k_{i}+|\mathbf{k}|}}{\left(t_{0} u_{i} q / t_{i} ; q\right)_{k_{i}+|\mathbf{k}|}} \\
& \times \prod_{i=1}^{n} \frac{\left(b u_{i}, t_{0}^{2} u_{i} q^{1+M} / b d t_{1} \cdots t_{n} ; q\right)_{k_{i}}}{\left(t_{0} u_{i} q / d, t_{0} u_{i} q^{1+M} ; q\right)_{k_{i}}} \cdot \frac{\left(d, q^{-M} ; q\right)_{|\mathbf{k}|}}{\left(t_{0} q / b, b d t_{1} \cdots t_{n} q^{-M} / t_{0} ; q\right)_{|\mathbf{k}|}} q^{|\mathbf{k}|}
\end{aligned}
$$

and

$$
\begin{aligned}
a_{\mathbf{l}}= & \frac{\left(t_{0} q / b d, t_{0} q / b t_{1} \cdots t_{n} ; q\right)_{M}}{\left(t_{0} q / b, t_{0} q / b d t_{1} \cdots t_{n} ; q\right)_{M}} \prod_{i=1}^{n} \frac{\left(t_{0} u_{i} q, t_{0} u_{i} q / d t_{i} ; q\right)_{M}}{\left(t_{0} u_{i} q / t_{i}, t_{0} u_{i} q / d ; q\right)_{M}} \\
& \times \prod_{1 \leq i<j \leq n}\left(u_{i} q^{l_{i}}-u_{j} q^{l_{j}}\right) \prod_{i, j=1}^{n} \frac{\left(t_{j} u_{i} / u_{j} ; q\right)_{l_{i}-l_{j}}}{\left(q u_{i} / u_{j} ; q\right)_{l_{i}-l_{j}}} \prod_{i=1}^{n} \frac{\left(d q^{-M} / t_{0} u_{i} ; q\right)_{|\mathbf{1}|-l_{i}}}{\left(d t_{i} q^{-M} / t_{0} u_{i} ; q\right)_{|\mathbf{1}|-l_{i}}} \\
& \times \prod_{i=1}^{n} \frac{\left(b u_{i}, t_{0}^{2} u_{i} q^{1+M} / b d t_{1} \cdots t_{n} ; q\right)_{l_{i}}}{\left(t_{0} u_{i} q / d t_{i}, t_{0} u_{i} q^{1+M} / t_{i} ; q\right)_{l_{i}}} \cdot \frac{\left(d, q^{-M} ; q\right)_{|\mathbf{1}|}}{\left(t_{0} q / b t_{1} \cdots t_{n}, b d q^{-M} / t_{0} ; q\right)_{|\mathbf{1}|}} q^{|\mathbf{|}|} \prod_{i=1}^{n} t_{i}^{-l_{i}},
\end{aligned}
$$

and $g_{\mathbf{k} \mathbf{l}}$ as in (A.9b). Therefore, we must have (A.4a) with the above sequences $a_{\mathbf{l}}$ and $b_{\mathbf{k}}$, and $f_{\mathbf{m k}}$ as in (A.9a). After the substitutions $t_{0} \mapsto t_{0} q^{-|\mathbf{k}|}, d \mapsto d q^{-|\mathbf{k}|}, u_{i} \mapsto u_{i} q^{-k_{i}}, m_{i} \mapsto m_{i}+k_{i}$, $i=1, \ldots, n, M \mapsto M+|\mathbf{k}|$, simplifications and subsequent relabelling $\left(m_{i} \mapsto k_{i}, i=1, \ldots, n\right)$, we obtain (4.1).

The following identity is obtained from Theorem 4.1 by a polynomial argument.

Corollary 4.2 (A multivariable terminating ${ }_{8} \phi_{7}$ summation). Let $a, b, c, d$, and $u_{1}, \ldots, u_{n}$ be indeterminate, let $m_{1}, \ldots, m_{n}$ be nonnegative integers. Then

$$
\begin{aligned}
& \sum_{\substack{0 \leq k_{i} \leq m_{i} \\
i=1, \ldots, n}} \prod_{i, j=1}^{n} \frac{\left(q^{1-m_{i}} u_{i} / u_{j} ; q\right)_{k_{i}}}{\left(q u_{i} / u_{j} ; q\right)_{k_{i}}} \prod_{1 \leq i<j \leq n} \frac{\left(q^{m_{j}} u_{i} / u_{j} ; q\right)_{k_{i}-k_{j}}}{\left(q^{1-m_{i}} u_{i} / u_{j} ; q\right)_{k_{i}-k_{j}}} \prod_{1 \leq i<j \leq n} \frac{1}{u_{i}-u_{j}} \\
& \times \operatorname{det}_{1 \leq i, j \leq n}\left[\left(u_{i} q^{k_{i}}\right)^{n-j}\left(1-q^{(j-n-1) m_{i}} \frac{1-a u_{i} q^{k_{i}+|\mathbf{m}|}}{1-a u_{i} q^{k_{i}+|\mathbf{m}|-m_{i}}} \prod_{s=1}^{n} \frac{u_{i} q^{k_{i}}-u_{s}}{u_{i} q^{k_{i}-m_{i}}-u_{s}}\right)\right] \\
& \times \frac{(c, d ; q)_{|\mathbf{k}|}}{\left(b c d q^{-|\mathbf{m}|} / a, a q / b ; q\right)_{|\mathbf{k}|}} \prod_{i=1}^{n} \frac{\left(a u_{i} q^{1+|\mathbf{m}|-m_{i}}, b u_{i}, a^{2} u_{i} q^{1+|\mathbf{m}|} / b c d ; q\right)_{k_{i}}}{\left(a u_{i} q^{1+|\mathbf{m}|}, a u_{i} q^{1+|\mathbf{m}|-m_{i}} / c, a u_{i} q^{1+|\mathbf{m}|-m_{i}} / d ; q\right)_{k_{i}}} \\
& \times \prod_{i=1}^{n} \frac{\left(c d q^{-|\mathbf{m}|} / a u_{i} ; q\right)_{|\mathbf{k}|-k_{i}}}{\left(c d q^{m_{i}-|\mathbf{m}|} / a u_{i} ; q\right)_{|\mathbf{k}|-k_{i}}} \cdot q^{|\mathbf{k}|+\sum_{i=1}^{n}\left((1-i) k_{i}+n_{i}\left[(i-1) k_{i}+\sum_{j=i+1}^{n} k_{j}\right]\right)} \\
& =\frac{(a q / b c, a q / b d ; q)_{|\mathbf{m}|}}{(a q / b, a q / b c d ; q)_{|\mathbf{m}|}} \prod_{i=1}^{n} \frac{\left(a u_{i} q^{1+|\mathbf{m}|-m_{i}}, a u_{i} q^{1+|\mathbf{m}|-m_{i}} / c d ; q\right)_{m_{i}}}{\left(a u_{i} q^{1+|\mathbf{m}|-m_{i}} / c, a u_{i} q^{1+|\mathbf{m}|-m_{i}} / d ; q\right)_{m_{i}}} .
\end{aligned}
$$

Proof. First we write the right hand-side of (4.1) as quotient of infinite products using (2.2). Then by the $t_{0}=a q^{|\mathbf{m}|}$ and $t_{i}=q^{m_{i}}, i=1, \ldots, n$, case of Theorem 3.6 it follows that the identity (4.2) holds for $c=q^{-M}$. By clearing out denominators in (4.2), we get a polynomial equation in $c$, which is true for $q^{-M}, M=0,1, \ldots$, and thus obtain an identity in $c$.

The following multivariable nonterminating ${ }_{6} \phi_{5}$ summation is obtained by letting $M \rightarrow \infty$ in Theorem 4.1 (while appealing to Tannery's theorem (cf. [7]) for justification of taking term-wise limits). 
Corollary 4.3 (A multivariable nonterminating ${ }_{6} \phi_{5}$ summation). Let $b, d, t_{0}, t_{1}, \ldots, t_{n}$, and $u_{1}, \ldots, u_{n}$ be indeterminate. Then

$$
\begin{aligned}
& \sum_{k_{1}, \ldots, k_{n} \geq 0} \prod_{i, j=1}^{n} \frac{\left(q u_{i} / t_{i} u_{j} ; q\right)_{k_{i}}}{\left(q u_{i} / u_{j} ; q\right)_{k_{i}}} \prod_{1 \leq i<j \leq n} \frac{\left(t_{j} u_{i} / u_{j} ; q\right)_{k_{i}-k_{j}}}{\left(q u_{i} / t_{i} u_{j} ; q\right)_{k_{i}-k_{j}}} \prod_{1 \leq i<j \leq n} \frac{1}{u_{i}-u_{j}} \\
& \times \operatorname{det}_{1 \leq i, j \leq n}\left[\left(u_{i} q^{k_{i}}\right)^{n-j}\left(1-t_{i}^{j-n-1} \frac{1-t_{0} u_{i} q^{k_{i}}}{1-t_{0} u_{i} q^{k_{i}} / t_{i}} \prod_{s=1}^{n} \frac{u_{i} q^{k_{i}}-u_{s}}{u_{i} q^{k_{i}} / t_{i}-u_{s}}\right)\right] q^{\sum_{i=1}^{n}(1-i) k_{i}} \\
& \times \prod_{i=1}^{n} \frac{\left(t_{0} u_{i} q / t_{i}, b u_{i} ; q\right)_{k_{i}}}{\left(t_{0} u_{i} q, t_{0} u_{i} q / d t_{i} ; q\right)_{k_{i}}} t_{i}^{i k_{i}-\sum_{j=1}^{i} k_{j}} \cdot \frac{(d ; q)_{|\mathbf{k}|}}{\left(t_{0} q / b t_{1} \cdots t_{n} ; q\right)_{|\mathbf{k}|}}\left(\frac{t_{0} q}{b d}\right)^{|\mathbf{k}|} \\
& =\frac{\left(t_{0} q / b, t_{0} q / b d t_{1} \cdots t_{n} ; q\right)_{\infty}}{\left(t_{0} q / b d, t_{0} q / b t_{1} \cdots t_{n} ; q\right)_{\infty}} \prod_{i=1}^{n} \frac{\left(t_{0} u_{i} q / t_{i}, t_{0} u_{i} q / d ; q\right)_{\infty}}{\left(t_{0} u_{i} q, t_{0} u_{i} q / d t_{i} ; q\right)_{\infty}},
\end{aligned}
$$

provided $\left|t_{0} q / b d\right|<1$.

The following identity is obtained from Corollary 4.3 by letting $d \rightarrow q^{-M}$.

Corollary 4.4 (A multivariable terminating ${ }_{6} \phi_{5}$ summation). Let $b, t_{0}, t_{1}, \ldots, t_{n}$, and $u_{1}, \ldots, u_{n}$ be indeterminate, let $M$ be a nonnegative integer. Then

$$
\begin{aligned}
& \sum_{\substack{k_{1}, \ldots, k_{n} \geq 0 \\
|\mathbf{k}| \leq M}} \prod_{i, j=1}^{n} \frac{\left(q u_{i} / t_{i} u_{j} ; q\right)_{k_{i}}}{\left(q u_{i} / u_{j} ; q\right)_{k_{i}}} \prod_{1 \leq i<j \leq n} \frac{\left(t_{j} u_{i} / u_{j} ; q\right)_{k_{i}-k_{j}}}{\left(q u_{i} / t_{i} u_{j} ; q\right)_{k_{i}-k_{j}}} \prod_{1 \leq i<j \leq n} \frac{1}{u_{i}-u_{j}} \\
& \quad \times \operatorname{det}_{1 \leq i, j \leq n}\left[( u _ { i } q ^ { k _ { i } } ) ^ { n - j } \left(1-t_{i}^{j-n-1} \frac{1-t_{0} u_{i} q^{k_{i}}}{1-t_{0} u_{i} q^{k_{i} / t_{i}}} \prod_{s=1}^{n} \frac{u_{i} q^{k_{i}}-u_{s}}{\left.\left.u_{i} q^{k_{i} / t_{i}-u_{s}}\right)\right] q^{\sum_{i=1}^{n}(1-i) k_{i}}}\right.\right. \\
& \quad \times \prod_{i=1}^{n} \frac{\left(t_{0} u_{i} q / t_{i}, b u_{i} ; q\right)_{k_{i}}}{\left(t_{0} u_{i} q, t_{0} u_{i} q^{1+M} / t_{i} ; q\right)_{k_{i}}} t^{i k_{i}-\sum_{j=1}^{i} k_{j}} \cdot \frac{\left(q^{-M} ; q\right)_{|\mathbf{k}|}}{\left(t_{0} q / b t_{1} \cdots t_{n} ; q\right)_{|\mathbf{k}|}}\left(\frac{t_{0} q^{1+M}}{b}\right)^{|\mathbf{k}|} \\
& \quad=\frac{\left(t_{0} q / b ; q\right)_{M}}{\left(t_{0} q / b t_{1} \cdots t_{n} ; q\right)_{M}} \prod_{i=1}^{n} \frac{\left(t_{0} u_{i} q / t_{i} ; q\right)_{M}}{\left(t_{0} u_{i} q ; q\right)_{M}} .
\end{aligned}
$$

Another multivariable terminating ${ }_{6} \phi_{5}$ summation theorem is obtained from Corollary 4.3 by letting $t_{i} \rightarrow q^{m_{i}}, i=1, \ldots, n$. We do not state it explicitly.

The last result in this section is, at this moment, only conjectural (which we however verified numerically by computer). We provide a heuristic (but not completely correct) derivation using the $C_{n}{ }_{8} \phi_{7}$ summation in Proposition 3.7 combined with the multivariable matrix inverse in Corollary A.6.

Conjecture 4.5 (A multivariable terminating ${ }_{8} \phi_{7}$ summation). Let $a, b, c$, $d$, and $u_{1}, \ldots, u_{n}$ be indeterminate, let $m_{1}, m_{2}, \ldots, m_{n}$ be nonnegative integers. Then

$$
\begin{aligned}
& \sum_{\substack{0 \leq k_{i} \leq m_{i} \\
i=1, \ldots, n}} \prod_{i, j=1}^{n} \frac{\left(q^{1-m_{i}} u_{i} / u_{j}, a u_{i} u_{j} q^{1+|\mathbf{m}|-m_{i}} ; q\right)_{k_{i}}\left(q^{m_{j}} u_{i} / u_{j} ; q\right)_{k_{i}-k_{j}}}{\left(q u_{i} / u_{j}, a u_{i} u_{j} q^{1+|\mathbf{m}|} ; q\right)_{k_{i}}\left(q u_{i} / u_{j} ; q\right)_{k_{i}-k_{j}}} \prod_{1 \leq i<j \leq n} \frac{u_{i} q^{k_{i}}-u_{j} q^{k_{j}}}{u_{i}-u_{j}} \\
& \quad \times \prod_{1 \leq i<j \leq n} \frac{\left(a u_{i} u_{j} q^{|\mathbf{m}|} ; q\right)_{k_{i}+k_{j}}}{\left(a u_{i} u_{j} q^{1+|\mathbf{m}|-m_{i}-m_{j}} ; q\right)_{k_{i}+k_{j}}} \cdot q^{|\mathbf{k}|+(n-1) \sum_{i=1}^{n} m_{i} k_{i}} \\
& \quad \times \prod_{1 \leq i<j \leq n}\left(u_{i}-u_{j}\right)^{-1}\left(1-q^{-|\mathbf{m}|} / a u_{i} u_{j}\right)^{-1} \prod_{i=1}^{n}\left(u_{i}+q^{-|\mathbf{m}|} / a u_{i}\right)^{-1}
\end{aligned}
$$




$$
\begin{aligned}
& \times \operatorname{det}_{1 \leq i, j \leq n}\left[\left(u_{i} q^{k_{i}}+q^{-k_{i}-m_{i}} / a u_{i}\right)^{n+1-j}-\left(u_{i} q^{k_{i}-m_{i}}+q^{-k_{i}+m_{i}-|\mathbf{m}|} / a u_{i}\right)^{n+1-j}\right. \\
& \left.\times \prod_{s=1}^{n} \frac{\left(u_{i} q^{k_{i}}-u_{s}\right)\left(1-q^{-k_{i}-|\mathbf{m}|} / a u_{i} u_{s}\right)}{\left(u_{i} q^{k_{i}-m_{i}}-u_{s}\right)\left(1-q^{-k_{i}+m_{i}-|\mathbf{m}|} / a u_{i} u_{s}\right)}\right] \\
& \times \prod_{i=1}^{n} \frac{\left(b u_{i}, c u_{i}, d u_{i}, a^{2} u_{i} q^{1+|\mathbf{m}|} / b c d ; q\right)_{k_{i}}}{\left(a u_{i} q^{1+|\mathbf{m}|-m_{i}} / b, a u_{i} q^{1+|\mathbf{m}|-m_{i}} / c, a u_{i} q^{1+|\mathbf{m}|-m_{i}} / d, b c d u_{i} q^{-m_{i}} / a ; q\right)_{k_{i}}} \\
& =\prod_{1 \leq i<j \leq n}\left(a u_{i} u_{j} q^{1+|\mathbf{m}|-m_{i}-m_{j}} ; q\right)_{m_{i}+m_{j}}^{-1} \prod_{i, j=1}^{n}\left(a u_{i} u_{j} q^{1+|\mathbf{m}|-m_{i}} ; q\right)_{m_{i}} \\
& (a q / b c, a q / b d, a q / c d ; q)_{|\mathbf{m}|} \\
& \times \frac{n}{\prod_{i=1}^{n}\left(a u_{i} q^{1+|\mathbf{m}|-m_{i}} / b, a u_{i} q^{1+|\mathbf{m}|-m_{i}} / c, a u_{i} q^{1+|\mathbf{m}|-m_{i}} / d, a q / b c d u_{i} ; q\right)_{m_{i}}}
\end{aligned}
$$

Heuristic derivation of Conjecture 4.5. We apply multidimensional inverse relations. We have (A.4b) by the $u_{i} \mapsto u_{i} q^{l_{i}}, M_{i} \mapsto M_{i}-l_{i}, i=1, \ldots, n$, case of Proposition 3.7, where

$$
\begin{aligned}
b_{\mathbf{k}}= & \prod_{1 \leq i<j \leq n} \frac{u_{i} q^{k_{i}}-u_{j} q^{k_{j}}}{u_{i}-u_{j}} \prod_{1 \leq i \leq j \leq n} \frac{1-a u_{i} u_{j} q^{k_{i}+k_{j}}}{1-a u_{i} u_{j}} \prod_{i, j=1}^{n} \frac{\left(u_{i} q^{-M_{j}} / u_{j} ; q\right)_{k_{i}-k_{j}}\left(a u_{i} u_{j} ; q\right)_{k_{i}+k_{j}}}{\left(q u_{i} / u_{j} ; q\right)_{k_{i}-k_{j}}\left(a u_{i} u_{j} q^{1+M_{i}} ; q\right)_{k_{i}+k_{j}}} \\
& \times \prod_{i=1}^{n} \frac{\left(b u_{i}, c u_{i}, d u_{i}, a^{2} u_{i} q^{1+|\mathbf{M}|} / b c d ; q\right)_{k_{i}}}{\left(a u_{i} q / b, a u_{i} q / c, a u_{i} q / d, b c d u_{i} q^{-|\mathbf{M}| / a ; q)_{k_{i}}}\right.} \cdot q^{|\mathbf{k}|}
\end{aligned}
$$

and

$$
\begin{aligned}
a_{\mathbf{l}}= & \prod_{1 \leq i<j \leq n}\left(a u_{i} u_{j} q^{1+l_{i}+l_{j}} ; q\right)_{M_{i}+M_{j}}^{-1} \prod_{i, j=1}^{n}\left(a u_{i} u_{j} q^{1+l_{i}+l_{j}} ; q\right)_{M_{i}} \\
& \times \frac{(a q / b c, a q / b d, a q / c d ; q)_{|\mathbf{M}|}}{\prod_{i=1}^{n}\left(a u_{i} q^{1+l_{i}} / b, a u_{i} q^{1+l_{i}} / c, a u_{i} q^{1+l_{i}} / d, a q^{\left.1+|\mathbf{M}|-M_{i}-l_{i} / b c d u_{i} ; q\right)_{M_{i}}}\right.} \\
& \times \prod_{1 \leq i<j \leq n} \frac{u_{i} q^{l_{i}}-u_{j} q^{l_{j}}}{u_{i}-u_{j}} \prod_{1 \leq i \leq j \leq n} \frac{1-a u_{i} u_{j} q^{l_{i}+l_{j}}}{1-a u_{i} u_{j}} \prod_{i, j=1}^{n} \frac{\left(u_{i} q^{-M_{j}} / u_{j} ; q\right)_{l_{i}-l_{j}}\left(a u_{i} u_{j} ; q\right)_{l_{i}+l_{j}}}{\left(q u_{i} / u_{j} ; q\right)_{l_{i}-l_{j}}\left(a u_{i} u_{j} q^{1+M_{i}} ; q\right)_{l_{i}+l_{j}}} \\
& \times \prod_{i=1}^{n} \frac{\left(b u_{i}, c u_{i}, d u_{i}, a^{2} u_{i} q^{1+|\mathbf{M}|} / b c d ; q\right)_{l_{i}}}{\left(a u_{i} q / b, a u_{i} q / c, a u_{i} q / d, b c d u_{i} q^{-|\mathbf{M}|} / a ; q\right)_{l_{i}}} \cdot q^{|\mathbf{1}|},
\end{aligned}
$$

and $g_{\mathbf{k l}}$ as in (A.10b) with $t_{i} \mapsto q^{-M_{i}}, i=1, \ldots, n$.

If we now consider (A.4a) with the above sequences $a_{\mathbf{l}}$ and $b_{\mathbf{k}}$, and $f_{\mathbf{m k}}$ as in (A.10a) with $t_{i} \mapsto q^{-M_{i}}, i=1, \ldots, n$, but do everywhere the replacements $M_{i} \mapsto-M_{i}, i=1, \ldots, n$ (the latter would require justification), we obtain, after the substitutions $a \mapsto a q^{|\mathbf{m}|}, u_{i} \mapsto u_{i} q^{-k_{i}}$, $m_{i} \mapsto m_{i}+k_{i}, i=1, \ldots, n$, simplifications and subsequent relabelling $\left(m_{i} \mapsto k_{i}, M_{i} \mapsto m_{i}\right.$, $i=1, \ldots, n)$, the identity in (4.3).

We hope that Conjecture 4.5 (assuming its correctness) will be useful in establishing explicit formulae related to $B_{n}, C_{n}$ or $D_{n}$ Macdonald polynomials.

\section{Hypergeometric specialization of $A_{n-1}$ Macdonald polynomials}

We show here that the Pieri formula, Theorem 2.1, and the (equivalent) recursion formula, Theorem 2.2, both can be viewed as multidimensional generalizations of the terminating verywell-poised ${ }_{6} \phi_{5}$ summation (3.3). This, together with the knowledge that the ${ }_{6} \phi_{5}$ summation 
has a nonterminating extension, see (3.4), eventually leads us in Section 7 to extend the family of Macdonald polynomials, indexed by partitions, to Macdonald symmetric functions, indexed by $n$-tuples of complex numbers.

Following Macdonald [33, p. 338], we consider for an indeterminate $u$ the homomorphism $\varepsilon_{u, t}:$ Sym $\rightarrow \mathbb{Q}(q, t)$, defined by

$$
\varepsilon_{u, t}\left(p_{r}\right)=\frac{1-u^{r}}{1-t^{r}},
$$

for each integer $r \geq 1$. In particular, if $u$ is replaced by $t^{n}$ one has

$$
\varepsilon_{t^{n}, t}\left(p_{r}\right)=\frac{1-t^{n r}}{1-t^{r}}=p_{r}\left(1, t, \ldots, t^{n-1}\right),
$$

and hence for any $f \in$ Sym

$$
\varepsilon_{t^{n}, t}(f)=f\left(1, t, \ldots, t^{n-1}\right),
$$

the "principal specialization" of $f$. (Another notation for the principal specialization is $u_{0}(f)[33$, p. 331], while in terms of plethystic notation it reads as $f\left[\frac{1-t^{n}}{1-t}\right]$; more generally, $\varepsilon_{u, t}(f)=f\left[\frac{1-u}{1-t}\right]$, relating to (5.1).) Since (recall (2.7))

$$
\varepsilon_{t^{n}, t} P_{\lambda}(X ; q, t)=t^{\sum_{i=1}^{n}(i-1) \lambda_{i}} \prod_{1 \leq i<j \leq n} \frac{\left(t^{j-i+1} ; q\right)_{\lambda_{i}-\lambda_{j}}}{\left(t^{j-i} ; q\right)_{\lambda_{i}-\lambda_{j}}},
$$

we have (see [33, p. 338, Eq. (6.17)])

$$
\varepsilon_{u, t} P_{\lambda}(X ; q, t)=t^{\sum_{i=1}^{n}(i-1) \lambda_{i}} \prod_{i=1}^{n} \frac{\left(u t^{1-i} ; q\right)_{\lambda_{i}}}{\left(t^{n+1-i} ; q\right)_{\lambda_{i}}} \prod_{1 \leq i<j \leq n} \frac{\left(t^{j-i+1} ; q\right)_{\lambda_{i}-\lambda_{j}}}{\left(t^{j-i} ; q\right)_{\lambda_{i}-\lambda_{j}}}
$$

where $n \geq l(\lambda)$. (We can take any such $n$. The homomorphism $\varepsilon_{u, t}$ in fact does not depend on $n$.) This follows from (5.2) by a simple polynomial argument. Both sides of (5.3) are polynomials in $u$ (with coefficients in $\mathbb{Q}(q, t)$ ) which agree for infinitely many values of $u$, namely whenever $u=t^{n}$ for any $n \geq l(\lambda)$, hence are identically equal.

Combining (2.5), (2.6) and (5.3), we have

$$
\begin{aligned}
& \varepsilon_{u, t} Q_{\lambda}(X ; q, t)=b_{\lambda}(q, t) \varepsilon_{u, t} P_{\lambda}(X ; q, t) \\
& =\prod_{i=1}^{n} \frac{\left(t^{n+1-i} ; q\right)_{\lambda_{i}}}{\left(q t^{n-i} ; q\right)_{\lambda_{i}}} \prod_{1 \leq i<j \leq n} \frac{\left(q t^{j-i}, t^{j-i} ; q\right)_{\lambda_{i}-\lambda_{j}}}{\left(t^{j-i+1}, q t^{j-i-1} ; q\right)_{\lambda_{i}-\lambda_{j}}} \\
& \times t^{\sum_{i=1}^{n}(i-1) \lambda_{i}} \prod_{i=1}^{n} \frac{\left(u t^{1-i} ; q\right)_{\lambda_{i}}}{\left(t^{n+1-i} ; q\right)_{\lambda_{i}}} \prod_{1 \leq i<j \leq n} \frac{\left(t^{j-i+1} ; q\right)_{\lambda_{i}-\lambda_{j}}}{\left(t^{j-i} ; q\right)_{\lambda_{i}-\lambda_{j}}} \\
& =t^{\sum_{i=1}^{n}(i-1) \lambda_{i}} \prod_{i=1}^{n} \frac{\left(u t^{1-i} ; q\right)_{\lambda_{i}}}{\left(q t^{n-i} ; q\right)_{\lambda_{i}}} \prod_{1 \leq i<j \leq n} \frac{\left(q t^{j-i} ; q\right)_{\lambda_{i}-\lambda_{j}}}{\left(q t^{j-i-1} ; q\right)_{\lambda_{i}-\lambda_{j}}},
\end{aligned}
$$

where $n \geq l(\lambda)$.

In view of the explicit evaluations (5.3) and (5.4), it is clear how useful the application of the homomorphism $\varepsilon_{u, t}$ is when applied to identities involving Macdonald polynomials, in particular for deriving multivariate formulae of hypergeometric type. In this respect (when being applied to Macdonald polynomials), we may consider $\varepsilon_{u, t}$ as a "hypergeometric specialization" (which 
is slightly misleading as $\varepsilon_{u, t}$ is actually not a specialization homomorphism for $u \neq t^{n}$; it can be regarded as an analytic continued specialization homomorphism though).

We mention that by applying $\varepsilon_{u, t}$ to the set $Y$ in the Cauchy formula [33, p. 324, Eq. (4.2)],

$$
\sum_{\lambda} P_{\lambda}(X ; q, t) Q_{\lambda}(Y ; q, t)=\prod_{i, j} \frac{\left(t x_{i} y_{j} ; q\right)_{\infty}}{\left(x_{i} y_{j} ; q\right)_{\infty}},
$$

Macdonald [33, p. 374, Eq. (4)] obtained a q-binomial theorem for multiple series of Macdonald polynomial argument. This identity became in fact the starting point in the development of a whole theory of identities for series of (classical $A_{n-1}$ ) Macdonald polynomial argument, see Kaneko [23, 24], Baker and Forrester [3], and Warnaar [54].

\subsection{Hypergeometric specialization of the Pieri formula}

We apply $\varepsilon_{u, t}$ to both sides of the Pieri formula in Theorem 2.1, utilizing (5.4). On the left-hand side we obtain

$$
\begin{aligned}
\varepsilon_{u, t} Q_{\left(\lambda_{1}, \ldots, \lambda_{n}\right)} \varepsilon_{u, t} Q_{\left(\lambda_{n+1}\right)}= & t^{\sum_{i=1}^{n}(i-1) \lambda_{i}} \prod_{i=1}^{n} \frac{\left(u t^{1-i} ; q\right)_{\lambda_{i}}}{\left(q t^{n-i} ; q\right)_{\lambda_{i}}} \\
& \times \prod_{1 \leq i<j \leq n} \frac{\left(q t^{j-i} ; q\right)_{\lambda_{i}-\lambda_{j}}}{\left(q t^{j-i-1} ; q\right)_{\lambda_{i}-\lambda_{j}}} \cdot \frac{(u ; q)_{\lambda_{n+1}}}{(q ; q)_{\lambda_{n+1}}} .
\end{aligned}
$$

Since

$$
\begin{aligned}
& \varepsilon_{u, t} Q_{\left(\lambda_{1}+\theta_{1}, \ldots, \lambda_{n}+\theta_{n}, \lambda_{n+1}-|\theta|\right)}=t t^{\left(\sum_{i=1}^{n}(i-1)\left(\lambda_{i}+\theta_{i}\right)\right)+n\left(\lambda_{n+1}-|\theta|\right)} \\
& \quad \times \prod_{i=1}^{n} \frac{\left(u t^{1-i} ; q\right)_{\lambda_{i}+\theta_{i}}}{\left(q t^{n+1-i} ; q\right)_{\lambda_{i}+\theta_{i}}} \cdot \frac{\left(u t^{-n} ; q\right)_{\lambda_{n+1}-|\theta|}}{(q ; q)_{\lambda_{n+1}-|\theta|}} \\
& \quad \times \prod_{1 \leq i<j \leq n} \frac{\left(q t^{j-i} ; q\right)_{\lambda_{i}+\theta_{i}-\lambda_{j}-\theta_{j}}}{\left(q t^{j-i-1} ; q\right)_{\lambda_{i}+\theta_{i}-\lambda_{j}-\theta_{j}}} \prod_{i=1}^{n} \frac{\left(q t^{n+1-i} ; q\right)_{\lambda_{i}+\theta_{i}-\lambda_{n+1}+|\theta|}}{\left(q t^{n-i} ; q\right)_{\lambda_{i}+\theta_{i}-\lambda_{n+1}+|\theta|}}
\end{aligned}
$$

we obtain on the right-hand side

$$
\begin{aligned}
& \sum_{\theta \in \mathbb{N}^{n}} \prod_{i=1}^{n} \frac{\left(t, q^{|\theta|+1+\lambda_{i}-\lambda_{n+1}} t^{n-i} ; q\right)_{\theta_{i}}}{\left(q, q^{|\theta|+\lambda_{i}-\lambda_{n+1}} t^{1+n-i} ; q\right)_{\theta_{i}}} \\
& \times \prod_{1 \leq i<j \leq n} \frac{\left(q^{\lambda_{i}-\lambda_{j}} t^{j-i+1}, q^{-\theta_{j}+1+\lambda_{i}-\lambda_{j}} t^{j-i-1} ; q\right)_{\theta_{i}}}{\left(q^{1+\lambda_{i}-\lambda_{j}} t^{j-i}, q^{-\theta_{j}+\lambda_{i}-\lambda_{j}} t^{j-i} ; q\right)_{\theta_{i}}} \cdot \varepsilon_{u, t} Q_{\left(\lambda_{1}+\theta_{1}, \ldots, \lambda_{n}+\theta_{n}, \lambda_{n+1}-|\theta|\right)} \\
& =\sum_{i=1}^{n+1}(i-1) \lambda_{i} \\
& \frac{\left(u t^{-n} ; q\right)_{\lambda_{n+1}}}{(q ; q)_{\lambda_{n+1}}} \prod_{i=1}^{n} \frac{\left(u t^{1-i} ; q\right)_{\lambda_{i}}}{\left(q t^{n+1-i} ; q\right)_{\lambda_{i}}} \frac{\left(q t^{n+1-i} ; q\right)_{\lambda_{i}-\lambda_{n+1}}}{\left(q t^{n-i} ; q\right)_{\lambda_{i}-\lambda_{n+1}}} \prod_{1 \leq i<j \leq n} \frac{\left(q t^{j-i} ; q\right)_{\lambda_{i}-\lambda_{j}}}{\left(q t^{j-i-1} ; q\right)_{\lambda_{i}-\lambda_{j}}} \\
& \times \sum_{\theta_{1}, \ldots, \theta_{n} \geq 0} \prod_{1 \leq i<j \leq n} \frac{\left(1-q^{\lambda_{i}-\lambda_{j}+\theta_{i}-\theta_{j}} t^{j-i}\right)}{\left(1-q^{\lambda_{i}-\lambda_{j}} t^{j-i}\right)} \prod_{i=1}^{n} \frac{\left(1-q^{\lambda_{i}-\lambda_{n+1}+\theta_{i}+|\theta|} t^{n+1-i}\right)}{\left(1-q^{\lambda_{i}-\lambda_{n+1}} t^{n+1-i}\right)} \\
& \times \prod_{i, j=1}^{n} \frac{\left(q^{\lambda_{i}-\lambda_{j}} t^{j-i+1} ; q\right)_{\theta_{i}}}{\left(q^{1+\lambda_{i}-\lambda_{j}} \prod^{j-i} ; q\right)_{\theta_{i}}} \prod_{i=1}^{n} \frac{\left(q^{\lambda_{i}-\lambda_{n+1}} t^{n+1-i} ; q\right)_{|\theta|}}{\left(q^{1+\lambda_{i}-\lambda_{n+1}} t^{n-i} ; q\right)_{|\theta|}} \\
& \times \prod_{i=1}^{n} \frac{\left(q^{\lambda_{i}} u t^{1-i} ; q\right)_{\theta_{i}}}{\left(q^{1+\lambda_{i}} t^{n+1-i} ; q\right)_{\theta_{i}}} \cdot \frac{\left(q^{-\lambda_{n+1}} ; q\right)_{|\theta|}}{\left(q^{1-\lambda_{n+1}} t^{n} / u ; q\right)_{|\theta|}}\left(\frac{q}{u}\right)^{|\theta|} \sum^{\sum_{i=1}^{n}(i-1) \theta_{i}} .
\end{aligned}
$$


If we equate the right-hand side expressions in (5.6) and (5.5), divide both sides by

$$
t^{\sum_{i=1}^{n+1}(i-1) \lambda_{i}} \frac{\left(u t^{-n} ; q\right)_{\lambda_{n+1}}}{(q ; q)_{\lambda_{n+1}}} \prod_{i=1}^{n} \frac{\left(u t^{1-i} ; q\right)_{\lambda_{i}}}{\left(q t^{n+1-i} ; q\right)_{\lambda_{i}}} \frac{\left(q t^{n+1-i} ; q\right)_{\lambda_{i}-\lambda_{n+1}}}{\left(q t^{n-i} ; q\right)_{\lambda_{i}-\lambda_{n+1}}} \prod_{1 \leq i<j \leq n} \frac{\left(q t^{j-i} ; q\right)_{\lambda_{i}-\lambda_{j}}}{\left(q t^{j-i-1} ; q\right)_{\lambda_{i}-\lambda_{j}}}
$$

and simplify, while rewriting the right-hand side using

$$
\frac{(u ; q)_{\lambda_{n+1}}}{\left(u t^{-n} ; q\right)_{\lambda_{n+1}}} \prod_{i=1}^{n} \frac{\left(q^{-\lambda_{i}} t^{i-n-1} ; q\right)_{\lambda_{n+1}}}{\left(q^{-\lambda_{i}} t^{i-n} ; q\right)_{\lambda_{n+1}}}=\frac{\left(q^{1-\lambda_{n+1}} / u ; q\right)_{\lambda_{n+1}}}{\left(q^{1-\lambda_{n+1}} t^{n} / u ; q\right)_{\lambda_{n+1}}} \prod_{i=1}^{n} \frac{\left(q^{1+\lambda_{i}-\lambda_{n+1}} t^{n+1-i} ; q\right)_{\lambda_{n+1}}}{\left(q^{1+\lambda_{i}-\lambda_{n+1}} t^{n-i} ; q\right)_{\lambda_{n+1}}}
$$

we obtain the following result:

$$
\begin{aligned}
& \sum_{\substack{\theta_{1}, \ldots, \theta_{n} \geq 0 \\
|\theta| \leq \lambda_{n+1}}} \prod_{1 \leq i<j \leq n} \frac{\left(q^{\lambda_{i}+\theta_{i}} t^{-i}-q^{\lambda_{j}+\theta_{j}} t^{-j}\right)}{\left(q^{\lambda_{i}} t^{-i}-q^{\lambda_{j}} t^{-j}\right)} \prod_{i=1}^{n} \frac{\left(1-q^{\lambda_{i}-\lambda_{n+1}+\theta_{i}+|\theta|} t^{n+1-i}\right)}{\left(1-q^{\lambda_{i}-\lambda_{n+1}} t^{n+1-i}\right)} \\
& \quad \times \prod_{i, j=1}^{n} \frac{\left(q^{\lambda_{i}-\lambda_{j}} t^{j-i+1} ; q\right)_{\theta_{i}}}{\left(q^{1+\lambda_{i}-\lambda_{j}} t^{j-i} ; q\right)_{\theta_{i}}} \prod_{i=1}^{n} \frac{\left(q^{\lambda_{i}-\lambda_{n+1}} t^{n+1-i} ; q\right)_{|\theta|}}{\left(q^{1+\lambda_{i}-\lambda_{n+1}} t^{n-i} ; q\right)_{|\theta|}} \\
& \quad \times \prod_{i=1}^{n} \frac{\left(q^{\lambda_{i}} u t^{1-i} ; q\right)_{\theta_{i}}}{\left(q^{1+\lambda_{i}} t^{n+1-i} ; q\right)_{\theta_{i}}} \cdot \frac{\left(q^{-\lambda_{n+1}} ; q\right)_{|\theta|}}{\left(q^{1-\lambda_{n+1}} t^{n} / u ; q\right)_{|\theta|}}\left(\frac{q}{u}\right)^{|\theta|} \\
& \quad=\frac{\left(q^{1-\lambda_{n+1}} / u ; q\right)_{\lambda_{n+1}}}{\left(q^{1-\lambda_{n+1}} t^{n} / u ; q\right)_{\lambda_{n+1}}} \prod_{i=1}^{n} \frac{\left(q^{1+\lambda_{i}-\lambda_{n+1}} t^{n+1-i} ; q\right)_{\lambda_{n+1}}}{\left(q^{1+\lambda_{i}-\lambda_{n+1}} t^{n-i} ; q\right)_{\lambda_{n+1}}} .
\end{aligned}
$$

Now, this identity (which is equivalent to the classical terminating ${ }_{6} \phi_{5}$ summation when $n=1$ ) is simply the special case of Milne's $A_{n-1}$ terminating ${ }_{6} \phi_{5}$ summation in Proposition 3.3, where $a \mapsto t, b \mapsto q^{\lambda_{n+1}} u t^{1-n}, c_{i} \mapsto t, u_{i} \mapsto q^{\lambda_{i}-\lambda_{n+1}} t^{n-i}(1 \leq i \leq n)$, and $M \mapsto \lambda_{n+1}$. In particular, observe that the $n+1$ indeterminates $a, c_{1}, \ldots, c_{n}$ all are substituted by $t$.

\subsection{Hypergeometric specialization of the recursion formula}

We apply $\varepsilon_{u, t}$ to both sides of the recursion formula in Theorem 2.2, utilizing (5.4). On the left-hand side we obtain

$$
\varepsilon_{u, t} Q_{\left(\lambda_{1}, \ldots, \lambda_{n+1}\right)}=t^{\sum_{i=1}^{n+1}(i-1) \lambda_{i}} \prod_{i=1}^{n+1} \frac{\left(u t^{1-i} ; q\right)_{\lambda_{i}}}{\left(q t^{n+1-i} ; q\right)_{\lambda_{i}}} \prod_{1 \leq i<j \leq n+1} \frac{\left(q t^{j-i} ; q\right)_{\lambda_{i}-\lambda_{j}}}{\left(q t^{j-i-1} ; q\right)_{\lambda_{i}-\lambda_{j}}} .
$$

Since

$$
\begin{aligned}
\varepsilon_{u, t} Q_{\left(\lambda_{n+1}-|\theta|\right)} \varepsilon_{u, t} Q_{\left(\lambda_{1}+\theta_{1}, \ldots, \lambda_{n}+\theta_{n}\right)}= & \frac{(u ; q)_{\lambda_{n+1}-|\theta|}}{(q ; q)_{\lambda_{n+1}-|\theta|}} \cdot t^{\sum_{i=1}^{n}(i-1)\left(\lambda_{i}+\theta_{i}\right)} \\
& \times \prod_{i=1}^{n} \frac{\left(u t^{1-i} ; q\right)_{\lambda_{i}+\theta_{i}}}{\left(q t^{n-i} ; q\right)_{\lambda_{i}+\theta_{i}}} \prod_{1 \leq i<j \leq n} \frac{\left(q t^{j-i} ; q\right)_{\lambda_{i}+\theta_{i}-\lambda_{j}-\theta_{j}}}{\left(q t^{j-i-1} ; q\right)_{\lambda_{i}+\theta_{i}-\lambda_{j}-\theta_{j}}},
\end{aligned}
$$

we obtain on the right-hand side

$$
\begin{aligned}
\sum_{\theta \in \mathbb{N}^{n}} & \prod_{i=1}^{n} t^{\theta_{i}} \frac{\left(q / t, q^{1+\lambda_{i}-\lambda_{n+1}} t^{n-i} ; q\right)_{\theta_{i}}}{\left(q, q^{1+\lambda_{i}-\lambda_{n+1}} t^{1+n-i} ; q\right)_{\theta_{i}}} \prod_{1 \leq i<j \leq n} \frac{\left(q^{1+\lambda_{i}-\lambda_{j}} t^{j-i-1}, q^{-\theta_{j}+\lambda_{i}-\lambda_{j}} t^{j-i+1} ; q\right)_{\theta_{i}}}{\left(q^{1+\lambda_{i}-\lambda_{j}} t^{j-i}, q^{-\theta_{j}+\lambda_{i}-\lambda_{j}} t^{j-i} ; q\right)_{\theta_{i}}} \\
& \times \prod_{1 \leq i<j \leq n} \frac{1}{\left(q^{\lambda_{i}-\lambda_{n+1}+\theta_{i}} t^{n-i}-q^{\lambda_{j}-\lambda_{n+1}+\theta_{j}} t^{n-j}\right)} \operatorname{det}_{1 \leq i, j \leq n}\left[\left(q^{\lambda_{i}-\lambda_{n+1}+\theta_{i}} t^{n-i}\right)^{n-j}\right.
\end{aligned}
$$




$$
\begin{aligned}
& \left.\times\left(1-t^{j-1} \frac{1-q^{\lambda_{i}-\lambda_{n+1}+\theta_{i}} t^{n-i+1}}{1-q^{\lambda_{i}-\lambda_{n+1}+\theta_{i}} t^{n-i}} \prod_{s=1}^{n} \frac{q^{\lambda_{s}-\lambda_{n+1}} t^{n-s}-q^{\lambda_{i}-\lambda_{n+1}+\theta_{i}} t^{n-i}}{q^{\lambda_{s}-\lambda_{n+1}} t^{n-s+1}-q^{\lambda_{i}-\lambda_{n+1}+\theta_{i}} t^{n-i}}\right)\right] \\
& \times \varepsilon_{u, t} Q_{\left(\lambda_{n+1}-|\theta|\right)} \varepsilon_{u, t} Q_{\left(\lambda_{1}+\theta_{1}, \ldots, \lambda_{n}+\theta_{n}\right)} \\
& =t^{\sum_{i=1}^{n}(i-1) \lambda_{i}} \frac{(u ; q)_{\lambda_{n+1}}}{(q ; q)_{\lambda_{n+1}}} \prod_{i=1}^{n} \frac{\left(u t^{1-i} ; q\right)_{\lambda_{i}}}{\left(q t^{n-i} ; q\right)_{\lambda_{i}}} \prod_{1 \leq i<j \leq n} \frac{\left(q t^{j-i} ; q\right)_{\lambda_{i}-\lambda_{j}}}{\left(q t^{j-i-1} ; q\right)_{\lambda_{i}-\lambda_{j}}} \\
& \times \sum_{\theta \in \mathbb{N}^{n}} \prod_{i, j=1}^{n} \frac{\left(q^{1+\lambda_{i}-\lambda_{j}} t^{j-i-1} ; q\right)_{\theta_{i}}}{\left(q^{1+\lambda_{i}-\lambda_{j}} t^{j-i} ; q\right)_{\theta_{i}}} \prod_{1 \leq i<j \leq n} \frac{\left(q^{\lambda_{i}-\lambda_{j}} t^{j-i+1} ; q\right)_{\theta_{i}-\theta_{j}}}{\left(q^{1+\lambda_{i}-\lambda_{j}} t^{j-i-1} ; q\right)_{\theta_{i}-\theta_{j}}} \\
& \times \prod_{1 \leq i<j \leq n} \frac{1}{\left(q^{\lambda_{i}-\lambda_{n+1}} t^{n-i}-q^{\lambda_{j}-\lambda_{n+1}} t^{n-j}\right)} \operatorname{det}_{1 \leq i, j \leq n}\left[\left(q^{\lambda_{i}-\lambda_{n+1}+\theta_{i}} t^{n-i}\right)^{n-j}\right. \\
& \left.\times\left(1-t^{j-1} \frac{1-q^{\lambda_{i}-\lambda_{n+1}+\theta_{i}} t^{n-i+1}}{1-q^{\lambda_{i}-\lambda_{n+1}+\theta_{i}} t^{n-i}} \prod_{s=1}^{n} \frac{q^{\lambda_{s}-\lambda_{n+1}} t^{n-s}-q^{\lambda_{i}-\lambda_{n+1}+\theta_{i}} t^{n-i}}{q^{\lambda_{s}-\lambda_{n+1}} t^{n-s+1}-q^{\lambda_{i}-\lambda_{n+1}+\theta_{i}} t^{n-i}}\right)\right] \\
& \times \prod_{i=1}^{n} \frac{\left(q^{1+\lambda_{i}-\lambda_{n+1}} t^{n-i}, q^{\lambda_{i}} u t^{1-i} ; q\right)_{\theta_{i}}}{\left(q^{1+\lambda_{i}-\lambda_{n+1}} t^{1+n-i}, q^{1+\lambda_{i}} t^{n-i} ; q\right)_{\theta_{i}}} \cdot \frac{\left(q^{-\lambda_{n+1}} ; q\right)_{|\theta|}}{\left(q^{1-\lambda_{n+1}} / u ; q\right)_{|\theta|}}\left(\frac{q t}{u}\right)^{|\theta|} q^{\sum^{n}(1-i) \theta_{i}} t^{\sum_{i=1}^{n} 2(i-1) \theta_{i}} .
\end{aligned}
$$

If we equate the right-hand side expressions in (5.8) and (5.7), divide both sides by

$$
t^{\sum_{i=1}^{n}(i-1) \lambda_{i}} \frac{(u ; q)_{\lambda_{n+1}}}{(q ; q)_{\lambda_{n+1}}} \prod_{i=1}^{n} \frac{\left(u t^{1-i} ; q\right)_{\lambda_{i}}}{\left(q t^{n-i} ; q\right)_{\lambda_{i}}} \prod_{1 \leq i<j \leq n} \frac{\left(q t^{j-i} ; q\right)_{\lambda_{i}-\lambda_{j}}}{\left(q t^{j-i-1} ; q\right)_{\lambda_{i}-\lambda_{j}}}
$$

and simplify, while rewriting the right-hand side using

$$
\begin{aligned}
& t^{n \lambda_{n+1}} \frac{\left(u t^{-n} ; q\right)_{\lambda_{n+1}}}{(u ; q)_{\lambda_{n+1}}} \prod_{i=1}^{n} \frac{\left(q t^{n-i} ; q\right)_{\lambda_{i}}\left(q t^{n+1-i} ; q\right)_{\lambda_{i}-\lambda_{n+1}}}{\left(q t^{n+1-i} ; q\right)_{\lambda_{i}}\left(q t^{n-i} ; q\right)_{\lambda_{i}-\lambda_{n+1}}} \\
& \quad=\frac{\left(q^{1-\lambda_{n+1}} t^{n} / u ; q\right)_{\lambda_{n+1}}^{n}}{\left(q^{1-\lambda_{n+1}} / u ; q\right)_{\lambda_{n+1}}} \frac{\left(q^{1+\lambda_{i}-\lambda_{n+1}} t^{n-i} ; q\right)_{\lambda_{n+1}}}{\left(q^{1+\lambda_{i}-\lambda_{n+1}} t^{n+1-i} ; q\right)_{\lambda_{n+1}}}
\end{aligned}
$$

we obtain the following result:

$$
\begin{aligned}
& \sum_{\theta \in \mathbb{N}^{n}} \prod_{i, j=1}^{n} \frac{\left(q^{1+\lambda_{i}-\lambda_{j}} t^{j-i-1} ; q\right)_{\theta_{i}}}{\left(q^{1+\lambda_{i}-\lambda_{j}} t^{j-i} ; q\right)_{\theta_{i}}} \prod_{1 \leq i<j \leq n} \frac{\left(q^{\lambda_{i}-\lambda_{j}} t^{j-i+1} ; q\right)_{\theta_{i}-\theta_{j}}}{\left(q^{1+\lambda_{i}-\lambda_{j}} t^{j-i-1} ; q\right)_{\theta_{i}-\theta_{j}}} \prod_{1 \leq i<j \leq n} \frac{1}{\left(q^{\lambda_{i}} t^{-i}-q^{\lambda_{j}} t^{-j}\right)} \\
& \times \operatorname{det}_{1 \leq i, j \leq n}\left[\left(q^{\lambda_{i}+\theta_{i}} t^{-i}\right)^{n-j}\left(1-t^{j-1} \frac{1-q^{\lambda_{i}-\lambda_{n+1}+\theta_{i}} t^{n-i+1}}{1-q^{\lambda_{i}-\lambda_{n+1}+\theta_{i}} t^{n-i}} \prod_{s=1}^{n} \frac{q^{\lambda_{s}} t^{-s}-q^{\lambda_{i}+\theta_{i}} t^{-i}}{q^{\lambda_{s}} t^{1-s}-q^{\lambda_{i}+\theta_{i}} t^{-i}}\right)\right]
\end{aligned}
$$

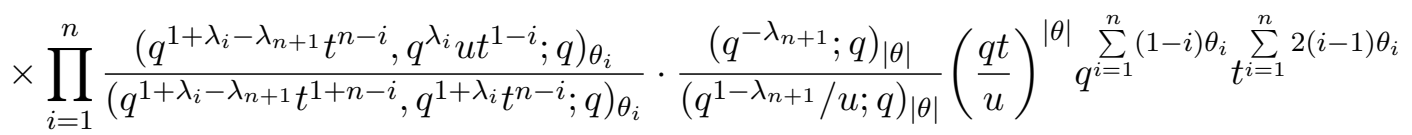

$$
\begin{aligned}
& =\frac{\left(q^{1-\lambda_{n+1}} t^{n} / u ; q\right)_{\lambda_{n+1}}}{\left(q^{1-\lambda_{n+1}} / u ; q\right)_{\lambda_{n+1}}} \prod_{i=1} \frac{\left(q^{1+\lambda_{i}-\lambda_{n+1}} t^{n-i} ; q\right)_{\lambda_{n+1}}}{\left(q^{1+\lambda_{i}-\lambda_{n+1}} t^{n+1-i} ; q\right)_{\lambda_{n+1}}} \text {. }
\end{aligned}
$$

Now, this identity (which is equivalent to the classical terminating ${ }_{6} \phi_{5}$ summation when $n=1$ ) is simply the special case of the multivariable terminating ${ }_{6} \phi_{5}$ summation in Corollary 4.4 , where $a \mapsto t, b \mapsto q^{\lambda_{n+1}} u t^{1-n}, c_{i} \mapsto t, u_{i} \mapsto q^{\lambda_{i}-\lambda_{n+1}} t^{n-i}(1 \leq i \leq n)$, and $M \mapsto \lambda_{n+1}$. In particular, observe that the $n+1$ indeterminates $a, c_{1}, \ldots, c_{n}$ all are substituted by $t$. 


\section{More basic hypergeometric identities involving Macdonald polynomials}

In the previous section we showed that the Pieri formula and the recursion formula, see Theorems 2.1 and 2.2 , both constitute (two different) multivariable terminating ${ }_{6} \phi_{5}$ summations which involve $A_{n-1}$ Macdonald polynomials. While nonterminating series are considered in Section 7, one can ask whether other important basic hypergeometric identities can be extended to the multivariate setting involving $A_{n-1}$ Macdonald polynomials. Concerning multivariate extensions of identities for non-very-well-poised basic hypergeometric series, we refer to the work of Kaneko [23, 24], Baker and Forrester [3], and Warnaar [54], where several identities are established that involve Macdonald polynomials playing the role of the argument of the respective series. In the very-well-poised case, which is investigated here, the $A_{n-1}$ Macdonald polynomials play the role of $q$-shifted factorials (to which they would reduce after principal specialization).

The main difference is the dimension of the series; in the $A_{n-1}$ setting the very-well-poised structure is implicit from taking the step $n \mapsto n+1$ to one higher dimension (see e.g. the proof of Proposition 3.3). Whereas for $n=1$ the $A_{n-1}$ Macdonald polynomials are simply monomials, in the $n=2$ case they are, in view of

$$
\begin{aligned}
P_{\left(\lambda_{1}, \lambda_{2}\right)}\left(x_{1}, x_{2} ; q, t\right) & =\left(x_{1} x_{2}\right)^{\lambda_{2}} P_{\left(\lambda_{1}-\lambda_{2}, 0\right)}\left(x_{1}, x_{2} ; q, t\right) \\
& =\left(x_{1} x_{2}\right)^{\lambda_{2}} \frac{(q ; q)_{\lambda_{1}-\lambda_{2}}}{(t ; q)_{\lambda_{1}-\lambda_{2}}} g_{\lambda_{1}-\lambda_{2}}\left(x_{1}, x_{2} ; q, t\right)
\end{aligned}
$$

(where we have used (2.9) and (2.11)), (multiples of the) continuous $q$-ultraspherical polynomials $g_{m}$. The latter are specific ${ }_{2} \phi_{1}$ series, see (7.1), which when principally specialized simplify to ratios of $q$-shifted factorials by virtue of the $\mathrm{Chu}$-Vandermonde summation theorem, the terminating special case of the $q$-Gauß summation in (3.2).

We mention that for the nonreduced irreducible root system $B C_{n}$ very-well-poised basic hypergeometric series identities involving Okounkov's [44] Macdonald interpolation polynomials or the more general Koornwinder-Macdonald polynomials (both which are of $B C_{n}$ type) have been established by Rains, see [45, Sec. 4] and [46, Sec. 4]. (The $A_{n-1}$ identities investigated here appear to be essentially different from related $B C_{n}$ identities found by Rains.)

One first question that arises is whether Jackson's terminating very-well-poised ${ }_{8} \phi_{7}$ summation (3.5) can be extended to the multivariate setting involving $A_{n-1}$ Macdonald polynomials. The answer is affirmative. Consider the Pieri formula in Theorem 2.1. It reads as

$$
Q_{\left(\lambda_{1}, \ldots, \lambda_{n}\right)} Q_{\left(\lambda_{n+1}\right)}=\sum_{\theta \in \mathbb{N}^{n}} d_{\theta_{1}, \ldots, \theta_{n}}^{(q, t)}\left(u_{1}, \ldots, u_{n}\right) Q_{\left(\lambda_{1}+\theta_{1}, \ldots, \lambda_{n}+\theta_{n}, \lambda_{n+1}-|\theta|\right)},
$$

with coefficients $d_{\theta_{1}, \ldots, \theta_{n}}\left(u_{1}, \ldots, u_{n}\right)$ defined in Subsection 2.1. We already know that equation (6.1), as it stands, represents a multivariable terminating very-well-poised ${ }_{6} \phi_{5}$ summation. The "trick" now is to restrict the set $X$ to $n$ variables, i.e. to take $|X|=n$. Due to property (2.8) the Macdonald polynomials appearing in the sum all vanish unless $|\theta|=\lambda_{n+1}$. We thus obtain

$$
Q_{\left(\lambda_{1}, \ldots, \lambda_{n}\right)} Q_{\left(\lambda_{n+1}\right)}=\sum_{\theta \in \mathbb{N}^{n},|\theta|=\lambda_{n+1}} d_{\theta_{1}, \ldots, \theta_{n}}^{(q, t)}\left(u_{1}, \ldots, u_{n}\right) Q_{\left(\lambda_{1}+\theta_{1}, \ldots, \lambda_{n}+\theta_{n}\right)},
$$

under the assumption $X=\left\{x_{1}, \ldots, x_{n}\right\}$. It turns out that when $\varepsilon_{u, t}$ is applied to both sides of the identity in (6.2) (in Section 5 we coined this as "hypergeometric specialization"), its $n=2$ case reduces to a variant of Jackson's ${ }_{8} \phi_{7}$ summation (3.5). In fact, if we replace $n$ by $n+1$ in (6.2), and substitute $\theta_{n+1}$ by $\lambda_{n+2}-\left(\theta_{1}+\cdots+\theta_{n}\right)$, we obtain an identity which, after application of $\varepsilon_{u, t}$, is essentially equivalent to Milne's $A_{n-1}$ extension of Jackson's ${ }_{8} \phi_{7}$ summation 
in Proposition 3.6. Therefore, we regard (6.2) as a multivariable extension of Jackson's verywell-poised ${ }_{8} \phi_{7}$ summation.

On the other hand, we were (so far) not able to deduce a multivariable ${ }_{8} \phi_{7}$ summation directly from the (nonspecialized) recursion formula in Theorem 2.2.

It would be interesting to find a multivariable extension of Bailey's terminating very-wellpoised ${ }_{10} \phi_{9}$ transformation formula (cf. [12, Eq. (III.28)]) involving $A_{n-1}$ Macdonald polynomials.

\section{$7 \quad$ Macdonald symmetric functions indexed by partitions with complex parts}

In this section we shall assume $|q|<1$. We use the recursion in Theorem 2.2 now to define Macdonald symmetric functions $Q_{\lambda}$ when $\lambda=\left(\lambda_{1}, \ldots, \lambda_{n}\right)$ is any sequence of complex numbers. One difficulty is to properly define the one row case. (Another issue is convergence, since we will be considering nonterminating sums.)

Kadell [21] used the classical definition of a Schur function in terms of a ratio of alternants to extend Schur functions to partitions with complex parts. (Independently, Danilov and Koshevoy [9] define "continuous Schur functions" by a multidimensional integral, with respect to a Lebesgue measure in $\mathbb{R}^{n(n-1) / 2}$, over all points of a particular polytope, and show by an inductive argument that these functions generalize the ratio of alternants formula for Schur functions. In fact, Danilov and Koshevoy's continuous Schur functions correspond exactly to Kadell's Schur functions indexed by partitions with real parts.) We want to stress that our proposed extension of Macdonald polynomials to complex parts (see below) does not reduce to Kadell's extension of Schur functions when $q=t$.

We shall begin with a finite number of variables, say $X=\left\{x_{0}, x_{1}, \ldots, x_{r}\right\}$. (For convenience, we start to label $X$ with 0 here). First, consider $m$ to be a nonnegative integer. By appealing to the $q$-binomial theorem in (3.1) it follows from taking coefficients of $u^{m}$ in the generating function in (2.4) that the one row Macdonald polynomials $Q_{(m)}(X ; q, t)=g_{m}(X ; q, t)$ can be written in the following explicit form:

$$
\begin{aligned}
g_{m} & =\sum_{\substack{k_{0}, \ldots, k_{r} \geq 0 \\
k_{0}+\cdots+k_{r}=m}} \prod_{i=0}^{r} \frac{(t ; q)_{k_{i}}}{(q ; q)_{k_{i}}} x_{i}^{k_{i}} \\
& =\sum_{\substack{k_{1}, \ldots, k_{r} \geq 0 \\
0 \leq k_{1}+\cdots+k_{r} \leq m}} \frac{(t ; q)_{m-\left(k_{1}+\cdots+k_{r}\right)}}{(q ; q)_{m-\left(k_{1}+\cdots+k_{r}\right)}} x_{0}^{m-\left(k_{1}+\cdots+k_{r}\right)} \prod_{i=1}^{r} \frac{(t ; q)_{k_{i}}}{(q ; q)_{k_{i}}} x_{i}^{k_{i}} .
\end{aligned}
$$

Although we do not need it here, we mention that for $r=1$ and $x_{0} x_{1}=1$, the $g_{m}$ reduce to the continuous $q$-ultraspherical polynomials of degree $m$ in the argument $\left(x_{0}+x_{1}\right) / 2$, considered in [12, Ex. 1.29].

We rewrite the above expression yet further, using the short notation $|\mathbf{k}|=k_{1}+\cdots+k_{r}$, and obtain the following explicit form:

$$
g_{m}=\frac{(t ; q)_{m}}{(q ; q)_{m}} x_{0}^{m} \sum_{\substack{k_{1}, \ldots, k_{r} \geq 0 \\ 0 \leq|\mathbf{k}| \leq m}} \frac{\left(q^{-m} ; q\right)_{|\mathbf{k}|}}{\left(q^{1-m} / t ; q\right)_{|\mathbf{k}|}} \prod_{i=1}^{r} \frac{(t ; q)_{k_{i}}}{(q ; q)_{k_{i}}}\left(\frac{q x_{i}}{t x_{0}}\right)^{k_{i}}
$$

Using the definition

$$
(a ; q)_{c}=\frac{(a ; q)_{\infty}}{\left(a q^{c} ; q\right)_{\infty}}
$$


(recall $|q|<1$ ) for any complex number $c$, we propose the following definition for a one row complex Macdonald function ${ }^{1}$ :

$$
Q_{(c)}=g_{c}=\frac{\left(t x_{0} ; q\right)_{c}}{(q ; q)_{c}} \frac{\left(q / t x_{0} ; q\right)_{-c}}{(q / t ; q)_{-c}} \sum_{k_{1}, \ldots, k_{r} \geq 0} \frac{\left(q^{-c} ; q\right)_{|\mathbf{k}|}}{\left(q^{1-c} / t ; q\right)_{|\mathbf{k}|}} \prod_{i=1}^{r} \frac{(t ; q)_{k_{i}}}{(q ; q)_{k_{i}}}\left(\frac{q x_{i}}{t x_{0}}\right)^{k_{i}}
$$

which converges (if the series does not terminate) for $\left|q x_{i} / t x_{0}\right|<1(1 \leq i \leq r)$.

We emphasize that (7.2) is not an analytic continuation of (7.1). In fact, (7.2) is neither analytic in $c$, nor in $q^{c}$ (in any domain around the origin). Another problem is that $g_{c}$ is not symmetric in all the $x_{i}(0 \leq i \leq r)$ but only in the last $r$ of the $x_{i}(1 \leq i \leq r)$. Indeed, already for $r=1$, the ${ }_{2} \phi_{1}$ transformation $g_{c}\left(x_{0}, x_{1}\right)=g_{c}\left(x_{1}, x_{0}\right)$ turns out to be false if $c$ is not an integer.

There are other possibilities to extend $g_{m}$ to complex numbers. By our definition (7.2), if $c$ is not a nonnegative integer and $q=t$ (the Schur function case), then we get $g_{c}=\infty$, which is different from Kadell's [21, Eq. (2.1)] $s_{(c)}\left(x_{0}\right)=x_{0}^{c}=e^{c \ln \left(x_{0}\right)}$. On the other hand, if we would have defined $g_{c}$ by (7.1) (for complex $m=c$; relaxing the restriction $|\mathbf{k}| \leq m$ of summation), our definition would have also not matched Kadell's in the $q=t$ case since after letting $q=t$ we would be left with a product of geometric series on the right-hand side. However, our particular choice of (7.2) is motivated by some nice properties, among which are (7.5) and (7.6).

Since $g_{c}\left(x_{0}, x_{1}, \ldots, x_{r}, 0\right)=g_{c}\left(x_{0}, x_{1}, \ldots, x_{r}\right)$, we may let $r \rightarrow \infty$ (compare to [33, p. 41]). In the following, we relax the restriction of $X$ being finite. Thus, we allow $r \in \mathbb{N} \cup \infty$.

Having provided a definition of $Q_{(c)}$ for any complex number $c$, it is now straightforward to extend Theorem 2.2 to Macdonald functions indexed by sequences of complex numbers. Let $\lambda=\left(\lambda_{1}, \ldots, \lambda_{n+1}\right)$ be an arbitrary sequence of complex numbers. We do not require $n+1 \leq|X|$. For any $1 \leq i \leq n+1$ define $u_{i}=q^{\lambda_{i}-\lambda_{n+1}} t^{n-i}$. Then $Q_{\lambda}(X ; q, t)$ is defined recursively by $(7.2)$ and

$$
Q_{\left(\lambda_{1}, \ldots, \lambda_{n+1}\right)}=\sum_{\theta \in \mathbb{N}^{n}} c_{\theta_{1}, \ldots, \theta_{n}}^{(q, t)}\left(u_{1}, \ldots, u_{n}\right) Q_{\left(\lambda_{n+1}-|\theta|\right)} Q_{\left(\lambda_{1}+\theta_{1}, \ldots, \lambda_{n}+\theta_{n}\right)},
$$

where $c_{\theta_{1}, \ldots, \theta_{n}}^{(q, t)}\left(u_{1}, \ldots, u_{n}\right)$ is the same as in Subsection 2.2 .

While the (finite) recursion in Theorem 2.2 was proved by inverting the known Pieri formula for Macdonald polynomials indexed by partitions, (7.3) now defines Macdonald functions in the general case. The expansion in (7.3) is in general infinite and converges (when it does not terminate) for $|q|,\left|q x_{i} / t x_{0}\right|<1(i \geq 1)$. As a matter of formal manipulations (using multidimensional inverse relations), the equivalence of Theorems 2.2 and 2.1 (Pieri formula) for these Macdonald functions of complex parts is immediate.

We do not know whether the complex $Q_{\lambda}$ form a family of orthogonal functions, nor whether they are eigenfunctions of the Macdonald operator (or some reasonable extension of this operator). These questions, among others, wait for investigation. What at all makes these extended objects interesting, then? In fact, it turns out that for these "complex Macdonald functions" a generalization of (at least) one of the so-called Macdonald (ex-)conjectures holds; in particular, they satisfy an explicit evaluation formula.

Observe that we have departed from the algebra of symmetric functions in $X=\left\{x_{0}, x_{1}, \ldots\right\}$. We are working in another algebra. It is necessary to provide some details.

It is convenient to make the following definitions. For a complex number $c$, introduce the following complex " $q, t$-powers" of $x$ :

$$
x^{[c]}=x^{[c ; q, t]}=\frac{(t x ; q)_{c}}{(t ; q)_{c}} \frac{(q / t x ; q)_{-c}}{(q / t ; q)_{-c}} .
$$

\footnotetext{
${ }^{1}$ We call these new objects functions as they are not anymore polynomials. However, note that the term Macdonald functions is also used to denote other objects, namely modified Bessel functions of the second kind [55]. (The latter have nothing to do with algebraist I.G. Macdonald who introduced the $P_{\lambda}(X ; q, t)$.)
} 
Note that if $k$ is an integer then $x^{[k]}=x^{k}$. More generally, $x^{[c+k]}=x^{[c]} x^{k}$.

Next, using " $q, t$-powers", extend the definition of power sums to complex numbers $c$ :

$$
p_{c}\left(x_{0}, x_{1}, \ldots\right)=\sum_{i \geq 0} x_{i}^{[c]} .
$$

(We will actually only need the one-variable case $p_{c}\left(x_{0}\right)=x_{0}^{[c]}$ here.) As usual, this definition may be extended to multiindices, $p_{\left(c_{1}, \ldots, c_{n}\right)}=p_{c_{1}} \cdots p_{c_{n}}$.

Let $\mathbb{C}$ denote the set of complex numbers. The algebra we are considering is (algebraically) generated by the uncountably infinite set of products

$$
\left\{p_{c}\left(x_{0}\right) p_{s}\left(x_{1}, x_{2}, \ldots\right) \mid c \in \mathbb{C}, s \in \mathbb{N}-0\right\}
$$

with coefficients in $\mathbb{C}((q, t))$. Note that the above set of products is not an algebraic basis as we do not have algebraic independence (e.g., $\left.\left(p_{c_{1}} p_{s_{1}}\right)\left(p_{c_{2}} p_{s_{2}}\right)=\left(p_{c_{1}} p_{s_{2}}\right)\left(p_{c_{2}} p_{s_{1}}\right)\right)$. We denote this algebra by $\widehat{\mathrm{Sym}}$.

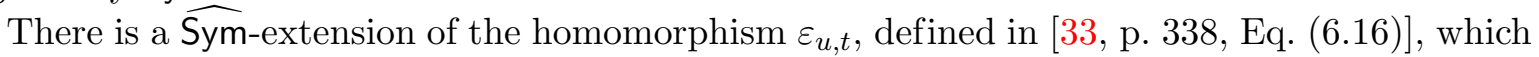
acts nicely on $Q_{\lambda}$. For an indeterminate $u$, define a homomorphism $\widehat{\varepsilon}_{u, t}: \widehat{\operatorname{Sym}} \rightarrow C((q, t))$ by

$$
\widehat{\varepsilon}_{u t, t}\left[p_{c}\left(x_{0}\right) p_{s}\left(x_{1}, x_{2}, \ldots\right)\right]=u^{[c]} \frac{1-u^{s}}{1-t^{s}}
$$

for each complex number $c$ and positive integer $s$. In particular, if $u$ is replaced by $t^{r}$, we have

$$
\widehat{\varepsilon}_{t^{r+1}, t}\left[p_{c}\left(x_{0}\right) p_{s}\left(x_{1}, x_{2}, \ldots\right)\right]=\left(t^{r}\right)^{[c]} \frac{1-t^{r s}}{1-t^{s}}=p_{c}\left(t^{r}\right) p_{s}\left(t^{r-1}, \ldots, t, 1\right)
$$

and hence for any $f \in \widehat{\mathrm{Sym}}$

$$
\widehat{\varepsilon}_{t^{r+1}, t}(f)=f\left(t^{r}, t^{r-1}, \ldots, t, 1\right) \text {. }
$$

(Compare this to the usual $\varepsilon_{t^{r+1}, t}(f)=f\left(t^{r}, t^{r-1}, \ldots, t, 1\right)$, for any $f \in$ Sym.)

We now have the evaluation formula

$$
\widehat{\varepsilon}_{u, t} Q_{\left(\lambda_{1}, \ldots, \lambda_{n}\right)}=\prod_{i=1}^{n} \frac{(u ; q)_{\lambda_{i}}}{\left(q t^{n-i} ; q\right)_{\lambda_{i}}} \frac{(q / u ; q)_{-\lambda_{i}}}{\left(q t^{i-1} / u ; q\right)_{-\lambda_{i}}} \prod_{1 \leq i<j \leq n} \frac{\left(q t^{j-i} ; q\right)_{\lambda_{i}-\lambda_{j}}}{\left(q t^{j-i-1} ; q\right)_{\lambda_{i}-\lambda_{j}}}
$$

where $\lambda_{i} \in \mathbb{C}$. We will present a pure basic hypergeometric proof (which reduces to a new proof of the usual analytic continued principal specialization formula for $Q_{\lambda}$ if $\lambda$ is a partition).

We proceed by induction on $n$. For $n=1$ we first consider $\widehat{\varepsilon}_{t^{r+1}, t} Q_{(c)}$ with $Q_{(c)}$ given in (7.2). By the $a_{i} \mapsto t, u_{i} \mapsto u^{i}, i=1, \ldots, n, b \mapsto q^{-c}$, and $c \mapsto q^{1-c} / t$ case of Proposition 3.5, we see that

$$
\begin{aligned}
\widehat{\varepsilon}_{t^{r+1}, t} Q_{(c)} & =\frac{\left(t^{r+1} ; q\right)_{c}}{(q ; q)_{c}} \frac{\left(q / t^{r+1} ; q\right)_{-c}}{(q / t ; q)_{-c}} \sum_{k_{1}, \ldots, k_{r} \geq 0} \frac{\left(q^{-c} ; q\right)_{|\mathbf{k}|}}{\left(q^{1-c} / t ; q\right)_{|\mathbf{k}|}} \prod_{i=1}^{r} \frac{(t ; q)_{k_{i}}}{(q ; q)_{k_{i}}}\left(q t^{-1-i}\right)^{k_{i}} \\
& =\frac{\left(t^{r+1} ; q\right)_{c}}{(q ; q)_{c}} \frac{\left(q / t^{r+1} ; q\right)_{-c}}{(q / t ; q)_{-c}} \frac{\left(q^{1-c} / t^{r+1}, q / t ; q\right)_{\infty}}{\left(q^{1-c} / t, q / t^{r+1} ; q\right)_{\infty}}=\frac{\left(t^{r+1} ; q\right)_{c}}{(q ; q)_{c}}
\end{aligned}
$$

which is exactly the $u=t^{r+1}$ case of the right-hand side of (7.5) for $n=1$. Since this holds for $r=0,1,2, \ldots$, by analytic continuation we may replace $t^{r+1}$ by $u$, which establishes the $n=1$ case of (7.5). For the inductive step, we assume (7.5) for partitions $\lambda$ with $l(\lambda) \leq n$. Apply $\widehat{\varepsilon}_{u, t}$ 
to both sides of the recursion formula (7.3) and use the inductive hypothesis to simplify the summand. We are done if we can show that the sum evaluates to

$$
\prod_{i=1}^{n+1} \frac{(u ; q)_{\lambda_{i}}}{\left(q t^{n+1-i} ; q\right)_{\lambda_{i}}} \frac{(q / u ; q)_{-\lambda_{i}}}{\left(q t^{i-1} / u ; q\right)_{-\lambda_{i}}} \prod_{1 \leq i<j \leq n+1} \frac{\left(q t^{j-i} ; q\right)_{\lambda_{i}-\lambda_{j}}}{\left(q t^{j-i-1} ; q\right)_{\lambda_{i}-\lambda_{j}}} .
$$

This, however, follows by an application of the multivariable ${ }_{6} \phi_{5}$ summation in Corollary 4.3 (after performing the substitutions $a \mapsto t, b \mapsto q^{\lambda_{n+1}} u t^{1-n}, c_{i} \mapsto t, u_{i} \mapsto q^{\lambda_{i}-\lambda_{n+1}} t^{n-i}, 1 \leq i \leq n$, and $M \mapsto \lambda_{n+1}$; compare with equation (5.9)).

There is a well-known duality formula for Macdonald polynomials (cf. [33, p. 332, Eq. (6.6)]),

$$
\frac{Q_{\lambda}\left(q^{\mu} t^{\delta}\right)}{Q_{\lambda}\left(t^{\delta}\right)}=\frac{Q_{\mu}\left(q^{\lambda} t^{\delta}\right)}{Q_{\mu}\left(t^{\delta}\right)}
$$

for partitions $\lambda$ and $\mu$ of length $\leq n$, where

$$
Q_{\lambda}\left(q^{\mu} t^{\delta}\right)=Q_{\lambda}\left(q^{\mu_{1}} t^{n-1}, q^{\mu_{2}} t^{n-2}, \ldots, q^{\mu_{n}}\right) .
$$

We do not know (at present) whether this relation still holds for arbitrary complex sequences $\lambda_{1}, \lambda_{2}, \ldots$, and $\mu_{1}, \mu_{2}, \ldots$ However, it does hold if the length of the partitions is one. Namely, if $\lambda=(c), \mu=(d)$ are one row complex partitions, we have

$$
\begin{aligned}
\frac{Q_{(c)}\left(q^{d} t, 1\right)}{Q_{(c)}(t, 1)} & =\frac{(q ; q)_{c}}{\left(t^{2} ; q\right)_{c}} \frac{\left(t x_{0} ; q\right)_{c}}{(q ; q)_{c}} \frac{\left(q / t x_{0} ; q\right)_{-c}}{(q / t ; q)_{-c}} \sum_{k \geq 0} \frac{\left(q^{-c}, t ; q\right)_{k}}{\left(q^{1-c} / t, q ; q\right)_{k}}\left(\frac{q^{1-d}}{t^{2}}\right)^{k} \\
& =\frac{\left(t x_{0} ; q\right)_{c}}{\left(t^{2} ; q\right)_{c}} \frac{\left(q / t x_{0} ; q\right)_{-c}}{(q / t ; q)_{-c}}{ }_{2} \phi_{1}\left[\begin{array}{c}
q^{-c}, t \\
q^{1-c} / t
\end{array} ; q, q^{1-d} / t^{2}\right]
\end{aligned}
$$

The duality is now an immediate consequence of the iterate of Heine's transformation [12, Eq. (III.2)],

$$
{ }_{2} \phi_{1}\left[\begin{array}{c}
a, b \\
c
\end{array} ; q, z\right]=\frac{(c / b, b z ; q)_{\infty}}{(c, z ; q)_{\infty}}{ }_{2} \phi_{1}\left[\begin{array}{c}
a b z / c, b \\
b z
\end{array} ;, \frac{c}{b}\right]
$$

valid for $\max (|z|,|c / b|)<1$.

To prevent possible misconception, we note that the well-known property valid for Macdonald polynomials indexed by partitions, $Q_{\lambda}\left(x_{1}, \ldots, x_{r}\right)=0$ if $l(\lambda)>r$ (see (2.5) and (2.8)), does not hold in the general complex case. For instance, if $X=\{x\}$ contains only one variable, then

$$
Q_{\left(\lambda_{1}, \ldots, \lambda_{n}\right)}(x)=\prod_{i=1}^{n} \frac{(t x ; q)_{\lambda_{i}}}{\left(q t^{n-i} ; q\right)_{\lambda_{i}}} \frac{(q / t x ; q)_{-\lambda_{i}}}{\left(q t^{i-2} ; q\right)_{-\lambda_{i}}} \prod_{1 \leq i<j \leq n} \frac{\left(q t^{j-i} ; q\right)_{\lambda_{i}-\lambda_{j}}}{\left(q t^{j-i-1} ; q\right)_{\lambda_{i}-\lambda_{j}}}
$$

where $\lambda_{i} \in \mathbb{C}$. This formula (which can be proved by induction, similar to the above proof of (7.5)) is indeed independent from the representation of $\lambda$, i.e. we may choose $\left(\lambda_{1}, \ldots, \lambda_{n}\right)=$ $\left(\lambda_{1}, \ldots, \lambda_{n}, 0, \ldots, 0\right)$, adding an arbitrary number of zeros at the end of sequence. It is clear from (7.6) that if $\lambda$ is a usual integer partition, then

$$
Q_{\left(\lambda_{1}, \ldots, \lambda_{n}\right)}(x)=\frac{(t ; q)_{\lambda_{1}}}{(q ; q)_{\lambda_{1}}} x^{\lambda_{1}} \delta_{\lambda_{2} 0} \ldots \delta_{\lambda_{n} 0}
$$

(where we were using (7.4) and $x^{[k]}=x^{k}$ for integer $k$ ).

In this section, we extended the Macdonald polynomials $Q_{\lambda}$ to arbitrary sequences $\lambda=$ $\left(\lambda_{1}, \ldots, \lambda_{n}\right)$ of complex numbers. To give such an extension for $P_{\lambda}$ one may simply invoke 
$Q_{\lambda}=b_{\lambda} P_{\lambda}$, see (2.5), with the known explicit expression of $b_{\lambda}=b_{\lambda}(q, t)$, extended to complex sequences $\lambda$. (This does not mean that we necessarily assume $b_{\lambda}=\left\langle Q_{\lambda}, Q_{\lambda}\right\rangle_{q, t}$ beforehand. Nevertheless, the latter equality should conjecturally still hold, for some suitable $\widehat{\text { Sym }}$-extension of the inner product $\langle,\rangle_{q, t}$. $)$ To utilize Theorem 2.3 to define $P_{\lambda}$ in the complex case does not make sense since the indexing partitions are given there in the form $\lambda=\left(1^{m_{1}}, 2^{m_{2}}, \ldots\right.$, $\left.(n+1)^{m_{n+1}}\right)$, and the multiplicities $m_{i}$ have no meaning for partitions with complex parts. (Already for compositions $\lambda$ one would run into trouble here.)

Again, we do not yet know whether the Macdonald functions for partitions with complex parts form a family of orthogonal functions. They very well may be orthogonal, possibly with respect to a $\widehat{\mathrm{Sym}}$ variant of the inner product in [33, p. 372, Eq. (9.10)]. In view of (7.5) and (7.6), some nice properties do exist, which provides some evidence that these new objects merit further investigation.

\section{A A multidimensional matrix inverse}

Here we state the multidimensional matrix inverse of [31, Th. 2.6], which happens to be so crucial for the results in this paper, and work out some special cases we need which we give as corollaries.

Let $\mathbb{Z}$ be the set of integers and $n$ some positive integer. For multi-integers $\mathbf{m}, \mathbf{k} \in \mathbb{Z}^{n}$, we write $\mathbf{m} \geq \mathbf{k}$ for $m_{i} \geq k_{i}$, for $i=1, \ldots, n$.

We say that an infinite $n$-dimensional matrix $F=\left(f_{\mathbf{m k}}\right)_{\mathbf{m}, \mathbf{k} \in \mathbb{Z}^{n}}$ is lower-triangular if $f_{\mathbf{m k}}=0$ unless $\mathbf{m} \geq \mathbf{k}$. When all $f_{\mathbf{k k}} \neq 0$, there exists a unique lower-triangular matrix $G=\left(g_{\mathbf{m k}}\right)_{\mathbf{m}, \mathbf{k} \in \mathbb{Z}^{n}}$, called the inverse of $F$, such that the following orthogonality relation holds:

$$
\sum_{\mathbf{m} \geq \mathbf{k}} f_{\mathbf{m k}} g_{\mathbf{k l}}=\delta_{\mathbf{m l}}, \quad \text { for all } \mathbf{m}, \mathbf{l} \in \mathbb{Z}^{n} .
$$

Clearly, as $F$ and $G$ are both lower-triangular, $F$ must then also be the inverse of $G$ and the dual relation,

$$
\sum_{\mathbf{m} \geq \mathbf{k}} g_{\mathbf{m k}} f_{\mathbf{k l}}=\delta_{\mathbf{m l}}, \quad \text { for all } \mathbf{m}, \mathbf{l} \in \mathbb{Z}^{n},
$$

must hold at the same time. Therefore, if $F$ and $G$ are infinite lower-triangular $n$-dimensional matrices and one of the relations (A.1) or (A.2) hold, we say that $F$ and $G$ are inverses of each other, or simply that $F$ and $G$ are mutually inverse.

It is immediate from the orthogonality relations (A.1) and (A.2) that if $F$ and $G$ are mutually inverse, the following two equations (a.k.a. "inverse relations"):

$$
\sum_{\mathbf{k} \in \mathbb{Z}^{n}} f_{\mathbf{m k}} a_{\mathbf{k}}=b_{\mathbf{m}}, \quad \text { for all } \mathbf{m}
$$

and

$$
\sum_{\mathbf{l} \in \mathbb{Z}^{n}} g_{\mathbf{k} \mathbf{l}} b_{\mathbf{l}}=a_{\mathbf{k}}, \quad \text { for all } \mathbf{k}
$$

are equivalent, provided both sides terminate or converge.

Similarly, the following two equations, where one sums over the first (instead of the second) multi-index of the respective matrices, are equivalent:

$$
\sum_{\mathbf{m} \in \mathbb{Z}^{n}} f_{\mathbf{m k}} a_{\mathbf{m}}=b_{\mathbf{k}}, \quad \text { for all } \mathbf{k},
$$


and

$$
\sum_{\mathbf{k} \in \mathbb{Z}^{n}} g_{\mathbf{k} \mathbf{l}} b_{\mathbf{k}}=a_{\mathbf{l}}, \quad \text { for all } \mathbf{l},
$$

again, provided both sides terminate or converge.

The inverse relations (A.3) or (A.4) are useful tools for proving identities. For instance, if (A.4b) holds for the infinite lower-triangular matrix $\left(g_{\mathbf{m k}}\right)_{\mathbf{m}, \mathbf{k} \in \mathbb{Z}^{n}}$ and infinite sequences $\left(b_{\mathbf{k}}\right)_{\mathbf{k} \in \mathbb{Z}^{n}}$ and $\left(a_{\mathbf{l}}\right)_{\mathbf{l} \in \mathbb{Z}^{n}}$, then (assuming one knows $F$, the inverse of $G$ ), the equation (A.4a) must automatically hold (subject to convergence). It is exactly this form of inverse relations which is being utilized in this paper.

The following matrix inversion was derived in [31, Th. 2.6].

Proposition A.1. Let $b$ be an indeterminate and $a_{i}(k), c_{i}(k)(k \in \mathbb{Z}, 1 \leq i \leq n)$ be arbitrary sequences of indeterminates. Then the infinite lower-triangular $n$-dimensional matrices $\left(f_{\mathbf{m k}}\right)_{\mathbf{m}, \mathbf{k} \in \mathbb{Z}^{n}}$ and $\left(g_{\mathbf{k} \mathbf{l}}\right)_{\mathbf{k}, \mathbf{l} \in \mathbb{Z}^{n}}$ are inverses of each other where

$$
\begin{aligned}
f_{\mathbf{m k}}= & \prod_{i=1}^{n} c_{i}\left(k_{i}\right)^{-1} \prod_{1 \leq i<j \leq n}\left(c_{i}\left(k_{i}\right)-c_{j}\left(k_{j}\right)\right)^{-1} \\
& \times \operatorname{det}_{1 \leq i, j \leq n}\left[c_{i}\left(m_{i}\right)^{n+1-j}-a_{i}\left(m_{i}\right)^{n+1-j} \frac{\left(c_{i}\left(m_{i}\right)-b / \prod_{s=1}^{n} c_{s}\left(k_{s}\right)\right)}{\left(a_{i}\left(m_{i}\right)-b / \prod_{s=1}^{n} c_{s}\left(k_{s}\right)\right)} \prod_{s=1}^{n} \frac{\left(c_{i}\left(m_{i}\right)-c_{s}\left(k_{s}\right)\right)}{\left(a_{i}\left(m_{i}\right)-c_{s}\left(k_{s}\right)\right)}\right] \\
& \times \prod_{i=1}^{n} \prod_{y_{i}=k_{i}+1}^{m_{i}}\left[\frac{\left(a_{i}\left(y_{i}\right)-b / \prod_{j=1}^{n} c_{j}\left(k_{j}\right)\right)}{\left(c_{i}\left(y_{i}\right)-b / \prod_{j=1}^{n} c_{j}\left(k_{j}\right)\right)} \prod_{j=1}^{n} \frac{\left(a_{i}\left(y_{i}\right)-c_{j}\left(k_{j}\right)\right)}{\left(c_{i}\left(y_{i}\right)-c_{j}\left(k_{j}\right)\right)}\right],
\end{aligned}
$$

and

$$
g_{\mathbf{k l}}=\prod_{i=1}^{n} \frac{\prod_{y_{i}=l_{i}+1}^{k_{i}}\left[\left(a_{i}\left(y_{i}\right)-b / \prod_{j=1}^{n} c_{j}\left(k_{j}\right)\right) \prod_{j=1}^{n}\left(a_{i}\left(y_{i}\right)-c_{j}\left(k_{j}\right)\right)\right]}{\prod_{y_{i}=l_{i}}^{k_{i}-1}\left[\left(c_{i}\left(y_{i}\right)-b / \prod_{j=1}^{n} c_{j}\left(k_{j}\right)\right) \prod_{j=1}^{n}\left(c_{i}\left(y_{i}\right)-c_{j}\left(k_{j}\right)\right)\right]} .
$$

Remark A.2. The above Proposition generalizes Krattenthaler's matrix inverse [26] which is obtained when $n=1$. Note that for $n=1$ the determinant in (A.5) reduces (after relabeling) to

$$
c_{m}-a_{m} \frac{\left(c_{m}-b / c_{k}\right)}{\left(a_{m}-b / c_{k}\right)} \frac{\left(c_{m}-c_{k}\right)}{\left(a_{m}-c_{k}\right)}=c_{m} \frac{\left(a_{m}-b / c_{m}\right)}{\left(a_{m}-b / c_{k}\right)} \frac{\left(a_{m}-c_{m}\right)}{\left(a_{m}-c_{k}\right)}
$$

and the matrix inverse in (A.5) (after relabeling) becomes

$$
\begin{aligned}
& f_{m k}=\frac{\left(b-a_{m} c_{m}\right)\left(a_{m}-c_{m}\right)}{\left(b-a_{k} c_{k}\right)\left(a_{k}-c_{k}\right)} \frac{\prod_{y=k}^{m-1}\left(a_{y}-b / c_{k}\right)\left(a_{y}-c_{k}\right)}{\prod_{y=k+1}^{m}\left(c_{y}-b / c_{k}\right)\left(c_{y}-c_{k}\right)}, \\
& g_{k l}=\frac{\prod_{y=l+1}^{k}\left(a_{y}-b / c_{k}\right)\left(a_{y}-c_{k}\right)}{\prod_{y=l}^{k-1}\left(c_{y}-b / c_{k}\right)\left(c_{y}-c_{k}\right)} .
\end{aligned}
$$


It is not difficult to see that this matrix inverse is actually equivalent to its $b \rightarrow \infty$ special case:

$$
\begin{gathered}
f_{m k}=\frac{\prod_{y=k}^{m-1}\left(a_{y}-c_{k}\right)}{\prod_{y=k+1}^{m}\left(c_{y}-c_{k}\right)}, \\
g_{k l}=\frac{\left(a_{l}-c_{l}\right)}{\left(a_{k}-c_{k}\right)} \frac{\prod_{y=l+1}^{k}\left(a_{y}-c_{k}\right)}{\prod_{y=l}^{k-1}\left(c_{y}-c_{k}\right)} .
\end{gathered}
$$

To recover (A.6) from (A.7), do the substitutions $a_{y} \mapsto a_{y}+b / a_{y}, c_{y} \mapsto c_{y}+b / c_{y}$, transfer some simple factors from one matrix to the other, and simplify.

Other multidimensional generalizations of Krattenthaler's matrix inverse were derived in [50, Th. 3.1], [27], and [51].

In [31, Th. 2.3] a matrix inverse slightly different to Proposition A.1, but equivalent to the latter, was given, which contains a determinant in the entries of $\left(g_{\mathbf{k} \mathbf{l}}\right)_{\mathbf{k}, \mathbf{l} \in \mathbb{Z}^{n}}$ instead of $\left(f_{\mathbf{m k}}\right)_{\mathbf{m}, \mathbf{k} \in \mathbb{Z}^{n}}$.

In the following we list a special case of Proposition A.1 which is derived in a procedure analogous to the derivation of (A.6) from (A.7). This result is itself a multidimensional generalization of the matrix inverse in (A.6).

Corollary A.3. Let $b$ be an indeterminate and $a_{i}(k), c_{i}(k)(k \in \mathbb{Z}, 1 \leq i \leq n)$ be arbitrary sequences of indeterminates. Then the infinite lower-triangular $n$-dimensional matrices $\left(f_{\mathbf{m k}}\right)_{\mathbf{m}, \mathbf{k} \in \mathbb{Z}^{n}}$ and $\left(g_{\mathbf{k} \mathbf{l}}\right)_{\mathbf{k}, \mathbf{l} \in \mathbb{Z}^{n}}$ are inverses of each other where

$$
\begin{aligned}
f_{\mathbf{m k}}= & \prod_{i=1}^{n} \frac{c_{i}\left(m_{i}\right)^{n}}{c_{i}\left(k_{i}\right)^{n}}\left(c_{i}\left(k_{i}\right)+b / c_{i}\left(k_{i}\right)\right)^{-1} \prod_{1 \leq i<j \leq n}\left[\left(1-b / c_{i}\left(k_{i}\right) c_{j}\left(k_{j}\right)\right)\left(c_{i}\left(k_{i}\right)-c_{j}\left(k_{j}\right)\right)\right]^{-1} \\
& \times \operatorname{det}_{1 \leq i, j \leq n}\left[\left(c_{i}\left(m_{i}\right)+b / c_{i}\left(m_{i}\right)\right)^{n+1-j}-\left(a_{i}\left(m_{i}\right)+b / a_{i}\left(m_{i}\right)\right)^{n+1-j}\right. \\
& \left.\times \prod_{s=1}^{n} \frac{\left(1-b / c_{i}\left(m_{i}\right) c_{s}\left(k_{s}\right)\right)\left(c_{i}\left(m_{i}\right)-c_{s}\left(k_{s}\right)\right)}{\left(1-b / a_{i}\left(m_{i}\right) c_{s}\left(k_{s}\right)\right)\left(a_{i}\left(m_{i}\right)-c_{s}\left(k_{s}\right)\right)}\right] \\
& \times \prod_{i, j=1}^{n} \prod_{y_{i}=k_{i}+1}^{m_{i}}\left[\frac{\left(a_{i}\left(y_{i}\right)-b / c_{j}\left(k_{j}\right)\right)\left(a_{i}\left(y_{i}\right)-c_{j}\left(k_{j}\right)\right)}{\left(c_{i}\left(y_{i}\right)-b / c_{j}\left(k_{j}\right)\right)\left(c_{i}\left(y_{i}\right)-c_{j}\left(k_{j}\right)\right)}\right]
\end{aligned}
$$

and

$$
g_{\mathbf{k} \mathbf{l}}=\prod_{i, j=1}^{n} \frac{\prod_{y_{i}=l_{i}+1}^{k_{i}}\left[\left(a_{i}\left(y_{i}\right)-b / c_{j}\left(k_{j}\right)\right)\left(a_{i}\left(y_{i}\right)-c_{j}\left(k_{j}\right)\right)\right]}{\prod_{y_{i}=l_{i}}^{k_{i}-1}\left[\left(c_{i}\left(y_{i}\right)-b / c_{j}\left(k_{j}\right)\right)\left(c_{i}\left(y_{i}\right)-c_{j}\left(k_{j}\right)\right)\right]} .
$$

Proof. In Proposition A.1, first let $b \rightarrow \infty$, then perform the substitutions $a_{i}\left(y_{i}\right) \mapsto a_{i}\left(y_{i}\right)+$ $b / a_{i}\left(y_{i}\right)$ and $c_{i}\left(y_{i}\right) \mapsto c_{i}\left(y_{i}\right)+b / c_{i}\left(y_{i}\right)$, for $1 \leq i \leq n$. Finally, transfer some factors from one matrix to the other.

We give two important special cases of the above multidimensional matrix inverses explicitly which involve the $q$-shifted factorials $(a ; q)_{k}$ defined in $(2.2)$. For $n=1$ the matrix inversions in Corollaries A.4 and A.6 both reduce to Bressoud's [6] matrix inverse, which he directly extracted from the terminating very-well-poised ${ }_{6} \phi_{5}$ summation (3.3). 
Corollary A.4. Let $t_{0}, t_{1}, \ldots, t_{n}$ and $u_{1}, \ldots, u_{n}$ be indeterminates. Then the infinite lowertriangular n-dimensional matrices $\left(f_{\mathbf{m k}}\right)_{\mathbf{m}, \mathbf{k} \in \mathbb{Z}^{n}}$ and $\left(g_{\mathbf{k} \mathbf{l}}\right)_{\mathbf{k}, \mathbf{l} \in \mathbb{Z}^{n}}$ are inverses of each other where

$$
\begin{aligned}
f_{\mathbf{m k}}= & q^{(n-1)(|\mathbf{k}|-|\mathbf{m}|)} \prod_{i=1}^{n} t_{i}^{n\left(m_{i}-k_{i}\right)} \prod_{1 \leq i<j \leq n}\left(u_{i} q^{k_{i}}-u_{j} q^{k_{j}}\right)^{-1} \\
& \times \operatorname{det}_{1 \leq i, j \leq n}\left[\left(u_{i} q^{m_{i}}\right)^{n-j}\left(1-t_{i}^{j-n-1} \frac{\left(1-t_{0} u_{i} q^{m_{i}+|\mathbf{k}|}\right)}{\left(1-t_{0} u_{i} q^{m_{i}+|\mathbf{k}|} / t_{i}\right)} \prod_{s=1}^{n} \frac{\left(u_{i} q^{m_{i}}-u_{s} q^{k_{s}}\right)}{\left(u_{i} q^{m_{i}} / t_{i}-u_{s} q^{k_{s}}\right)}\right)\right] \\
& \times \prod_{i=1}^{n} \frac{\left(t_{0} u_{i} q^{1+k_{i}+|\mathbf{k}|} / t_{i} ; q\right)_{m_{i}-k_{i}}}{\left(t_{0} u_{i} q^{1+k_{i}+|\mathbf{k}|} ; q\right)_{m_{i}-k_{i}}} \prod_{i, j=1}^{n} \frac{\left(q^{1+k_{i}-k_{j}} u_{i} / t_{i} u_{j} ; q\right)_{m_{i}-k_{i}}}{\left(q^{1+k_{i}-k_{j}} u_{i} / u_{j} ; q\right)_{m_{i}-k_{i}}}
\end{aligned}
$$

and

$$
\begin{aligned}
g_{\mathbf{k} \mathbf{l}}= & \prod_{i, j=1}^{n} \frac{\left(q u_{i} / u_{j} ; q\right)_{k_{i}-k_{j}}\left(t_{j} u_{i} / u_{j} ; q\right)_{l_{i}-l_{j}}}{\left(t_{j} u_{i} / u_{j} ; q\right)_{k_{i}-k_{j}}\left(q u_{i} / u_{j} ; q\right)_{l_{i}-l_{j}}} \\
& \times \prod_{i=1}^{n} \frac{\left(t_{0} u_{i} q^{1+l_{i}+|\mathbf{k}|} / t_{i} ; q\right)_{k_{i}-l_{i}}}{\left(t_{0} u_{i} q^{l_{i}+|\mathbf{k}|} ; q\right)_{k_{i}-l_{i}}} \prod_{i, j=1}^{n} \frac{\left(q^{l_{i}-l_{j}} t_{j} u_{i} / u_{j} ; q\right)_{k_{i}-l_{i}}}{\left(q^{1+l_{i}-l_{j}} u_{i} / u_{j} ; q\right)_{k_{i}-l_{i}}} .
\end{aligned}
$$

Remark A.5. The $t_{i}=t, i=0,1, \ldots, n$ case of Corollary A.4 is equivalent to Theorem 2.7 of [31].

Proof of Corollary A.4. We specialize Proposition A.1 by letting $b \mapsto t_{0}^{-1} \prod_{j=1}^{n} u_{j}, a_{i}\left(y_{i}\right) \mapsto$ $u_{i} q^{y_{i}} / t_{i}$, and $c_{i}\left(y_{i}\right) \mapsto u_{i} q^{y_{i}}$, for $1 \leq i \leq n$, and rewrite the expressions employing $q$-shifted factorial notation. We finally multiply $f_{\mathbf{m k}}$ by $\prod_{i=1}^{n}\left(q / t_{i}\right)^{n\left(k_{i}-m_{i}\right)}$ and $g_{\mathbf{k} \mathbf{l}}$ by $\prod_{i=1}^{n}\left(q / t_{i}\right)^{n\left(l_{i}-k_{i}\right)}$.

Corollary A.6. Let $t_{0}, t_{1}, \ldots, t_{n}$ and $u_{1}, \ldots, u_{n}$ be indeterminates. Then the infinite lowertriangular n-dimensional matrices $\left(f_{\mathbf{m k}}\right)_{\mathbf{m}, \mathbf{k} \in \mathbb{Z}^{n}}$ and $\left(g_{\mathbf{k} \mathbf{l}}\right)_{\mathbf{k}, \mathbf{l} \in \mathbb{Z}^{n}}$ are inverses of each other where

$$
\begin{aligned}
f_{\mathbf{m k}}= & \prod_{i=1}^{n} t_{i}^{n\left(m_{i}-k_{i}\right)}\left(u_{i} q^{k_{i}}+q^{-k_{i}} / a u_{i}\right)^{-1} \prod_{1 \leq i<j \leq n}\left[\left(u_{i} q^{k_{i}}-u_{j} q^{k_{j}}\right)\left(1-q^{-k_{i}-k_{j}} / a u_{i} u_{j}\right)\right]^{-1} \\
& \times \operatorname{det}_{1 \leq i, j \leq n}\left[\left(u_{i} q^{m_{i}}+q^{-m_{i}} / a u_{i}\right)^{n+1-j}-\left(u_{i} q^{m_{i}} / t_{i}+t_{i} q^{-m_{i}} / a u_{i}\right)^{n+1-j}\right. \\
& \left.\times \prod_{s=1}^{n} \frac{\left(1-q^{-m_{i}-k_{s}} / a u_{i} u_{s}\right)\left(u_{i} q^{m_{i}}-u_{s} q^{k_{s}}\right)}{\left(1-t_{i} q^{-m_{i}-k_{s}} / a u_{i} u_{s}\right)\left(u_{i} q^{m_{i}} / t_{i}-u_{s} q^{k_{s}}\right)}\right] \\
& \times \prod_{i, j=1}^{n} \frac{\left(q^{1+k_{i}-k_{j}} u_{i} / t_{i} u_{j}, a u_{i} u_{j} q^{1+k_{i}+k_{j}} / t_{i} ; q\right)_{m_{i}-k_{i}}}{\left(q^{1+k_{i}-k_{j}} u_{i} / u_{j}, a u_{i} u_{j} q^{1+k_{i}+k_{j}} ; q\right)_{m_{i}-k_{i}}}
\end{aligned}
$$

and

$$
\begin{aligned}
g_{\mathbf{k l}}= & \prod_{i, j=1}^{n} \frac{\left(q u_{i} / u_{j} ; q\right)_{k_{i}-k_{j}}\left(a u_{i} u_{j} q / t_{j} ; q\right)_{k_{i}+k_{j}}\left(t_{j} u_{i} / u_{j} ; q\right)_{l_{i}-l_{j}}\left(a u_{i} u_{j} ; q\right)_{l_{i}+l_{j}}}{\left(t_{j} u_{i} / u_{j} ; q\right)_{k_{i}-k_{j}}\left(a u_{i} u_{j} ; q\right)_{k_{i}+k_{j}}\left(q u_{i} / u_{j} ; q\right)_{l_{i}-l_{j}}\left(a u_{i} u_{j} q / t_{j} ; q\right)_{l_{i}+l_{j}}} \\
& \times \prod_{i, j=1}^{n} \frac{\left(q^{l_{i}-l_{j}} t_{j} u_{i} / u_{j}, a u_{i} u_{j} q^{l_{i}+l_{j}} ; q\right)_{k_{i}-l_{i}}}{\left(q^{1+l_{i}-l_{j}} u_{i} / u_{j}, a u_{i} u_{j} q^{l_{i}+l_{j}} / t_{j} ; q\right)_{k_{i}-l_{i}}}
\end{aligned}
$$


Proof. We specialize Theorem A.3 by letting $b \mapsto 1 / a, a_{i}\left(y_{i}\right) \mapsto u_{i} q^{y_{i}} / t_{i}$, and $c_{i}\left(y_{i}\right) \mapsto u_{i} q^{y_{i}}$, for $1 \leq i \leq n$, and rewrite the expressions employing $q$-shifted factorial notation. We finally multiply $f_{\mathbf{m k}}$ by $\prod_{i=1}^{n}\left(q / t_{i}\right)^{n\left(k_{i}-m_{i}\right)}$ and $g_{\mathbf{k l}}$ by $\prod_{i=1}^{n}\left(q / t_{i}\right)^{n\left(l_{i}-k_{i}\right)}$.

\section{Acknowledgements}

I would like to thank Michel Lassalle for getting me involved into Macdonald polynomials (especially concerning the issues related to matrix inversion and explicit expressions) and his encouragement. I would also like to express my sincere gratitude to the organizers of the "Workshop on Jack, Hall-Littlewood and Macdonald Polynomials" (ICMS, Edinburgh, September 23-26, 2003) for inviting me to participate in that very stimulating workshop. Among them, I am especially indebted to Vadim Kuznetsov whose interest in explicit formulae for Macdonald polynomials served as an inspiration for the present work.

The author was partly supported by FWF Austrian Science Fund grants P17563-N13, and S9607 (the second is part of the Austrian National Research Network "Analytic Combinatorics and Probabilistic Number Theory").

\section{References}

[1] Andrews G.E., $q$-series: their development and application in analysis, number theory, combinatorics, physics and computer algebra, CBMS Regional Conference Lectures Series, Vol. 66, Amer. Math. Soc., Providence, RI, 1986.

[2] Andrews G.E., Askey R., Roy R., Special functions, Encyclopedia of Mathematics and Its Applications, Vol. 71, Cambridge University Press, Cambridge, 1999.

[3] Baker T.H., Forrester P.J., Transformation formulas for multivariable basic hypergeometric series, Meth. Appl. Anal. 6 (1999), 147-164, math.QA/9803146.

[4] Bhatnagar G., A multivariable view of $q$-series, in Special Functions and Differential Equations, Editors K. Srinivasa Rao, R. Jagannathan, G. Vanden Berghe and J. Van der Jeugt, Proceedings of a Workshop, WSSF'97 (January 13-24, 1997, Madras, India), Allied Publ., New Delhi, 1998, 25-30.

[5] Bhatnagar G., Milne S.C., Generalized bibasic hypergeometric series and their $U(n)$ extensions, Adv. Math. 131 (1997), 188-252.

[6] Bressoud D.M., A matrix inverse, Proc. Amer. Math. Soc. 88 (1983), 446-448.

[7] Bromwich T.J.l'A., An introduction to the theory of infinite series, 2nd ed., Macmillan, London, 1949.

[8] Cauchy A.-L., Mémoire sur les fonctions dont plusieurs valeurs sont liées entre elles par une équation linéaire, et sur diverses transformations de produits composés d'un nombre indéfini de facteurs, C. R. Acad. Sci. Paris XVII (1843), 523; Oeuvres de Cauchy, $1^{\text {re }}$ série, T. VIII, Gauthier-Villars, Paris, 1893, 42-50.

[9] Danilov V., Koshevoy G., Continuous combinatorics, Preprint, 2005, 12 pages.

[10] Denis R.Y., Gustafson R.A., An $S U(n) q$-beta integral transformation and multiple hypergeometric series identities, SIAM J. Math. Anal. 23 (1992), 552-561.

[11] Frenkel I.B., Turaev V.G., Elliptic solutions of the Yang-Baxter equation and modular hypergeometric functions, in The Arnold-Gelfand Mathematical Seminars, Editors V.I. Arnold, I.M. Gelfand, V.S. Retakh and M. Smirnov, Birkhäuser, Boston, 1997, 171-204.

[12] Gasper G., Rahman M., Basic hypergeometric series, 2nd ed., Encyclopedia of Mathematics and Its Applications, Vol. 96, Cambridge University Press, Cambridge, 2004.

[13] Gustafson R.A., Multilateral summation theorems for ordinary and basic hypergeometric series in $U(n)$, SIAM J. Math. Anal. 18 (1987), 1576-1596.

[14] Gustafson R.A., The Macdonald identities for affine root systems of classical type and hypergeometric series very well-poised on semi-simple Lie algebras, in Ramanujan International Symposium on Analysis (December 26-28, 1987, Pune, India), Editor N.K. Thakare, 1989, 187-224.

[15] Heine E., Untersuchungen über die Reihe ..., J. Reine Angew. Math. 34 (1847), 285-328. 
[16] Holman W.J. III, Summation theorems for hypergeometric series in U(n), SIAM J. Math. Anal. 11 (1980), $523-532$.

[17] Holman W.J. III, Biedenharn L.C., Louck J.D., On hypergeometric series well-poised in $S U(n), S I A M J$. Math. Anal. 7 (1976), 529-541.

[18] Ismail M.E.H., Classical and quantum orthogonal polynomials in one variable, Encyclopedia of Mathematics and its Applications, Vol. 98, Cambridge University Press, Cambridge, 2005.

[19] Jackson F.H., Summation of q-hypergeometric series, Messenger of Math. 57 (1921), 101-112.

[20] Jing N.H., Józefiak T., A formula for two-row Macdonald functions, Duke Math. J. 67 (1992), $377-385$.

[21] Kadell K., The Schur functions for partitions with complex parts, Contemp. Math. 254 (2000), $247-270$.

[22] Kajihara Y., Noumi M., Raising operators of row type for Macdonald polynomials, Compos. Math. 120 (2000), 119-136, math.QA/9803151.

[23] Kaneko J., q-Selberg integrals and Macdonald polynomials, Ann. Sci. École Norm. Sup. 29 (1996), 583-637.

[24] Kaneko J., A ${ }_{1} \Psi_{1}$ summation theorem for Macdonald polynomials, Ramanujan J. 2 (1998), 379-386.

[25] Koornwinder T.H., Self-duality for $q$-ultraspherical polynomials associated with root system $A_{n}$, unpublished handwritten manuscript, 1988, 17 pages.

Available at http://remote.science.uva.nl/ thk/art/informal/dualmacdonald.pdf.

[26] Krattenthaler C., A new matrix inverse, Proc. Amer. Math. Soc. 124 (1996), 47-59.

[27] Krattenthaler C., Schlosser M.J., A new multidimensional matrix inverse with applications to multiple $q$-series, Discrete Math. 204 (1999), 249-279.

[28] Lassalle M., Explicitation des polynômes de Jack et de Macdonald en longueur trois, C. R. Acad. Sci. Paris Sér. I Math. 333 (2001), 505-508.

[29] Lassalle M., Une $q$-spécialisation pour les fonctions symétriques monomiales, Adv. Math. 162 (2001), 217242, math.CO/0004019.

[30] Lassalle M., A short proof of generalized Jacobi-Trudi expansions for Macdonald polynomials, Contemp. Math. 417 (2006), 271-280, math.CO/0401032.

[31] Lassalle M., Schlosser M.J., Inversion of the Pieri formula for Macdonald polynomials, Adv. Math. 202 (2006), 289-325, math.CO/0402127.

[32] Macdonald I.G., A new class of symmetric functions, Sém. Lothar. Combin. 20 (1988), Art. B20a, 41 pages.

[33] Macdonald I.G., Symmetric functions and hall polynomials, 2nd ed., Clarendon Press, Oxford, 1995.

[34] Macdonald I.G., Symmetric functions and orthogonal polynomials, Dean Jacqueline B. Lewis Memorial Lectures presented at Rutgers University, New Brunswick, NJ, University Lecture Series, Vol. 12, Amer. Math. Soc., Providence, RI, 1998.

[35] Milne S.C., An elementary proof of the Macdonald identities for $A_{\ell}^{(1)}$, Adv. Math. 57 (1985), 34-70.

[36] Milne S.C., Basic hypergeometric series very well-poised in $U(n)$, J. Math. Anal. Appl. 122 (1987), 223-256.

[37] Milne S.C., Multiple $q$-series and $U(n)$ generalizations of Ramanujan's ${ }_{1} \psi_{1}$ sum, in Ramanujan Revisited, Editors G.E. Andrews et al., Academic Press, New York, 1988, 473-524.

[38] Milne S.C., The multidimensional ${ }_{1} \Psi_{1}$ sum and Macdonald identities for $A_{l}^{(1)}$, in Theta Functions Bowdoin (1987), Editors L. Ehrenpreis and R.C. Gunning, Proc. Sympos. Pure Math. 49 (1989), 323-359.

[39] Milne S.C., A q-analog of a Whipple's transformation for hypergeometric series in $U(n)$, Adv. Math. 108 (1994), 1-76.

[40] Milne S.C., Balanced ${ }_{3} \phi_{2}$ summation theorems for $U(n)$ basic hypergeometric series, Adv. Math. 131 (1997), 93-187.

[41] Milne S.C., Transformations of $U(n+1)$ multiple basic hypergeometric series, in Physics and Combinatorics, Editors A.N. Kirillov, A. Tsuchiya and H. Umemura, Proceedings of the Nagoya 1999 International Workshop (August 23-27, 1999, Nagoya University, Japan), World Scientific, Singapore, 2001, 201-243.

[42] Milne S.C., Lilly G.M., Consequences of the $A_{l}$ and $C_{l}$ Bailey transform and Bailey lemma, Discrete Math. 139 (1995), 319-346, math.CA/9204236.

[43] Milne S.C., Newcomb J.W., U(n) very-well-poised ${ }_{10} \phi_{9}$ transformations, J. Comput. Appl. Math. 68 (1996), 239-285.

[44] Okounkov A., BC-type interpolation Macdonald polynomials and binomial formula for Koornwinder polynomials, Transform. Groups 3 (1998), 181-207, q-alg/9611011. 


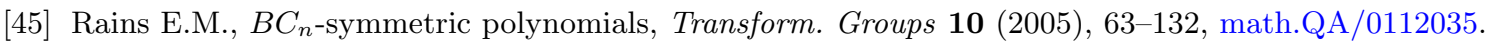

[46] Rains E.M., $B C_{n}$-symmetric Abelian functions, Duke Math. J. 135 (2006), 99-180, math.CO/0402113.

[47] Rogers R.J., Third memoir on the expansion of certain infinite products, Proc. London Math. Soc. 26 (1894), 15-32.

[48] Rosengren H., Elliptic hypergeometric series on root systems, Adv. Math. 181 (2004), 417-447, math.CA/0207046.

[49] Rosengren H., Reduction formulas for Karlsson-Minton-type hypergeometric functions, Constr. Approx. 20 (2004), 525-548, math.CA/0202232.

[50] Schlosser M.J., Multidimensional matrix inversions and $A_{r}$ and $D_{r}$ basic hypergeometric series, Ramanujan J. 1 (1997), 243-274.

[51] Schlosser M.J., A new multidimensional matrix inversion in $A_{r}$, Contemp. Math. 254 (2000), 413-432.

[52] Spiridonov V.P., Elliptic hypergeometric functions, Dr.Sc. Thesis, JINR, Dubna, Russia, 2004, 218 pages.

[53] Stanton D., An elementary approach to the Macdonald identities, in $q$-Series and Partitions, Editor D. Stanton, The IMA Volumes in Mathematics and Its Applications, Vol. 18, Springer-Verlag, 1989, 139-150.

[54] Warnaar S.O., q-Selberg integrals and Macdonald polynomials, Ramanujan J. 10 (2005), 237-268.

[55] Watson G.N., A treatise on the theory of Bessel functions, 2nd ed., Cambridge University Press, Cambridge, 1966. 